\title{
Inexpensive Microscopy for Introductory Laboratory Courses
}

\section{Citation}

Peidle, Joseph, Chris Stokes, Robert Hart, Melissa Franklin, Ronald Newburgh, Joon Pahk, Wolfgang Rueckner, and Aravi Samuel. 2009. Inexpensive microscopy for introductory laboratory courses. American Journal of Physics 77(10): 931-938.

\section{Published Version}

doi:10.1119/1.3153502

\section{Permanent link}

http://nrs.harvard.edu/urn-3:HUL.InstRepos:10403686

\section{Terms of Use}

This article was downloaded from Harvard University's DASH repository, and is made available under the terms and conditions applicable to Other Posted Material, as set forth at http:// nrs.harvard.edu/urn-3:HUL.InstRepos:dash.current.terms-of-use\#LAA

\section{Share Your Story}

The Harvard community has made this article openly available.

Please share how this access benefits you. Submit a story.

\section{Accessibility}




\title{
APPARATUS AND DEMONSTRATION NOTES
}

Frank L. H. Wolfs, Editor

Department of Physics and Astronomy, University of Rochester, Rochester, New York 14627

\begin{abstract}
This department welcomes brief communications reporting new demonstrations, laboratory equipment, techniques, or materials of interest to teachers of physics. Notes on new applications of older apparatus, measurements supplementing data supplied by manufacturers, information which, while not new, is not generally known, procurement information, and news about apparatus under development may be suitable for publication in this section. Neither the American Journal of Physics nor the Editors assume responsibility for the correctness of the information presented.

Manuscripts should be submitted using the web-based system that can be accessed via the American Journal of Physics home page, http://www.kzoo.edu/ajp/ and will be forwarded to the ADN editor for consideration.
\end{abstract}

\section{Inexpensive microscopy for introductory laboratory courses}

\author{
Joseph Peidle, Chris Stokes, Robert Hart, Melissa Franklin, Ronald Newburgh, \\ Joon Pahk, Wolfgang Rueckner, and Aravi Samuel \\ Harvard University, Cambridge, Massachusetts 02138
}

(Received 8 August 2008; accepted 22 May 2009)

\begin{abstract}
We present an inexpensive apparatus for bright field and fluorescence microscopy with video capture, suitable for introductory laboratory courses. Experiments on Brownian motion and the Boltzmann distribution of suspended particles in a gravitational field are described. The Boltzmann constant is measured in three ways, and the results fall within 15\% of the accepted value. (C) 2009 American Association of Physics Teachers.
\end{abstract}

[DOI: 10.1119/1.3153502]

\section{INTRODUCTION}

This paper describes an apparatus designed primarily for life science and premedical students. With this apparatus one can study Brownian motion, fluorescence microscopy, and the distribution of particles in sediment. It has the virtue of avoiding the black box nature of many undergraduate laboratory experiments. For example, when studying Brownian motion, the student actually sees the motion of polystyrene microspheres and appreciates the random nature of their displacements. Modern technology enters through the use of a digital camera and a computer. However, these devices aid the student and do not obscure the meaning of the data.

The apparatus does not need to be expensive. Using existing computers and vintage microscopes, we have been able to achieve a cost per unit of approximately $\$ 300$. The optional fluorescence attachment can be built for an additional $\$ 600$.

\section{PEDAGOGICAL MOTIVATION}

\section{A. Brownian motion}

Transport mechanisms within cells and locomotion of unicellular organisms take place in a regime governed by diffusion and Brownian motion. Living in the world of high Reynolds numbers, humans develop no intuition for what the life of a cell is like. We don't notice the thermal motion of air molecules or the random buffeting of water molecules when we swim. Students of the microscopic world, however, should study these statistical processes for a realistic understanding of the behavior of unicellular organisms.

Brownian motion was first examined experimentally and measured quantitatively in 1909 by Perrin. ${ }^{1}$ Recent papers ${ }^{2-4}$ discussed modern versions of Perrin's experiment to determine Avogadro's number or, equivalently, the Boltzmann constant. Perrin based his experiment on Einstein's theory of Brownian motion, ${ }^{5}$ specifically the equation for the mean square displacement of particles in suspension. This experiment can be readily adapted to advanced laboratory courses.

With the current apparatus, first-year students measure the mean square displacement of $1 \mu \mathrm{m}$ polystyrene spheres suspended in water and calculate the Boltzmann constant. Optional experiments include varying the diameter or density of the spheres or the viscosity of the aqueous solution. Students can also observe Brownian motion in biological samples such as yeast, tongue scrapings, and fat globules in milk.

\section{B. Fluorescence microscopy}

Any student interested in biological imaging will encounter fluorescence microscopy. The modern biologist who wants to find specific cells or molecules within a sample has but to choose the appropriate fluorescent tags. Only the tagged objects will be seen, absorbing photons at the excitation wavelength and releasing photons at the slightly longer emission wavelength. For many biologists, the fluorescence microscope is a "black box" that generates desired pictures, 
maps of colorfully labeled tags within their samples. The principles of wavelength-specific light sources, filters, and mirrors that enable fluorescence microscopes to work will demystify these devices for students who want to understand how their tools work.

With the current apparatus, students can disassemble the fluorescence attachment and measure the spectral properties of the LED used for illumination, the filters, and the fluorescent microspheres.

\section{Boltzmann distribution}

The Boltzmann distribution is used to describe a wide variety of classical systems such as the distribution of molecular speeds in a gas, the variation in pressure with elevation in the Earth's atmosphere, and the susceptibility of paramagnetic material. ${ }^{6}$ The height distribution of microspheres, settled near the bottom of an aqueous solution, also conforms to the Boltzmann distribution.

With the current apparatus, students measure the number density of microspheres as a function of height above the glass surface. Fluorescence microscopy is especially well suited for this measurement since the spheres above or below the focal plane appear dim compared to those in the focal plane.

\section{APPARATUS}

\section{A. Bright field microscope}

An optical microscope, configured for a viewer's eye, produces a final virtual image with a two-lens system. The objective lens produces a real intermediate image, which in turn is magnified by the eyepiece and viewed by the eye. If one desires to view the image on a computer screen rather than by eye, the real image produced by the objective may be projected directly onto a CCD plane. Since the pixels of a computer monitor are much larger than those of a CCD camera, a "digital magnification" substitutes for the magnification of the eyepiece.

In the present implementation, iMac computers and iSight cameras were used. The iMac LCD display pixels measure approximately $250 \mu \mathrm{m}$ on a side, while the camera pixels are approximately $5 \mu \mathrm{m}$, producing a digital magnification of about 50. $40 \times$ NA 0.65 objectives were used for a total magnification of 2000. The pixels were calibrated using a stage micrometer with $10 \mu \mathrm{m}$ tick marks, and it was not necessary to know the digital magnification precisely. LOGGER PRO software from Vernier Software and Technology Corporation was used to capture time-lapse videos.

The iSight cameras were modified by removing the lens and disabling the autofocus mechanism. ${ }^{7}$ A preferable method would be to purchase bare CCD cameras directly. Appendix A contains a list of inexpensive cameras suitable for our application.

We successfully used both the Bausch and Lomb (c. 1960) and the Spencer (c. 1920) microscopes shown in Fig. 1. The Spencer microscopes were preferred due to the reproducibil-

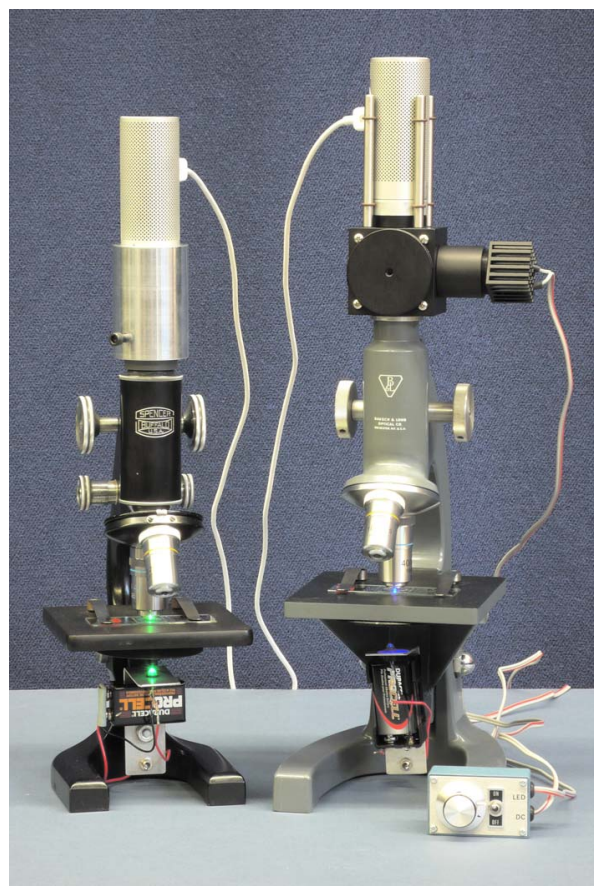

Fig. 1. (Color online) The Spencer (left) and the Bausch and Lomb (right) microscopes. The Spencer microscope is fitted with an iSight camera using a simple aluminum coupler. The eyepiece lens tube of the Bausch and Lomb microscope has been cut to compensate for the additional height of the fluorescence attachment.

ity of the fine-focus control. They are also easy to restore to working condition by cleaning the dovetails and lubricating the fine-focus mechanism. A simple machined aluminum tube coupled the camera to the microscope lens tube. Since standard microscope objectives are optimized to produce an image at $16 \mathrm{~cm}$, the camera should be mounted as close as possible to the top of the lens tube.

Illumination was provided by replacing the microscope mirrors with LEDs. A luminous intensity of the order of a few candelas is sufficient. We constructed ${ }^{8}$ simple circuits consisting of a battery, an LED, and a current-limiting resistor. The LED provides spatially coherent, narrow-band illumination, which makes it possible to observe diffraction patterns around each microsphere. The narrow-band illumination increases the depth of field compared to what can be obtained with diffuse illumination. This makes it easier to track particles as they diffuse in three dimensions. If directional illumination is not desired, a piece of paper can be taped under the microscope stage to act as a diffuser. Heating the sample, a concern with incandescent lamps, was insignificant with LED illumination. Shorter visible wavelengths, in the range from yellow to blue, were preferred.

Sample preparation techniques are important. Samples with significant evaporation will display unwanted convection currents. During the laboratory session the students can prepare samples that last for several hours, or the instructors can prepare sealed samples that last for days to months. Details of sample preparation are provided in Appendix B. 


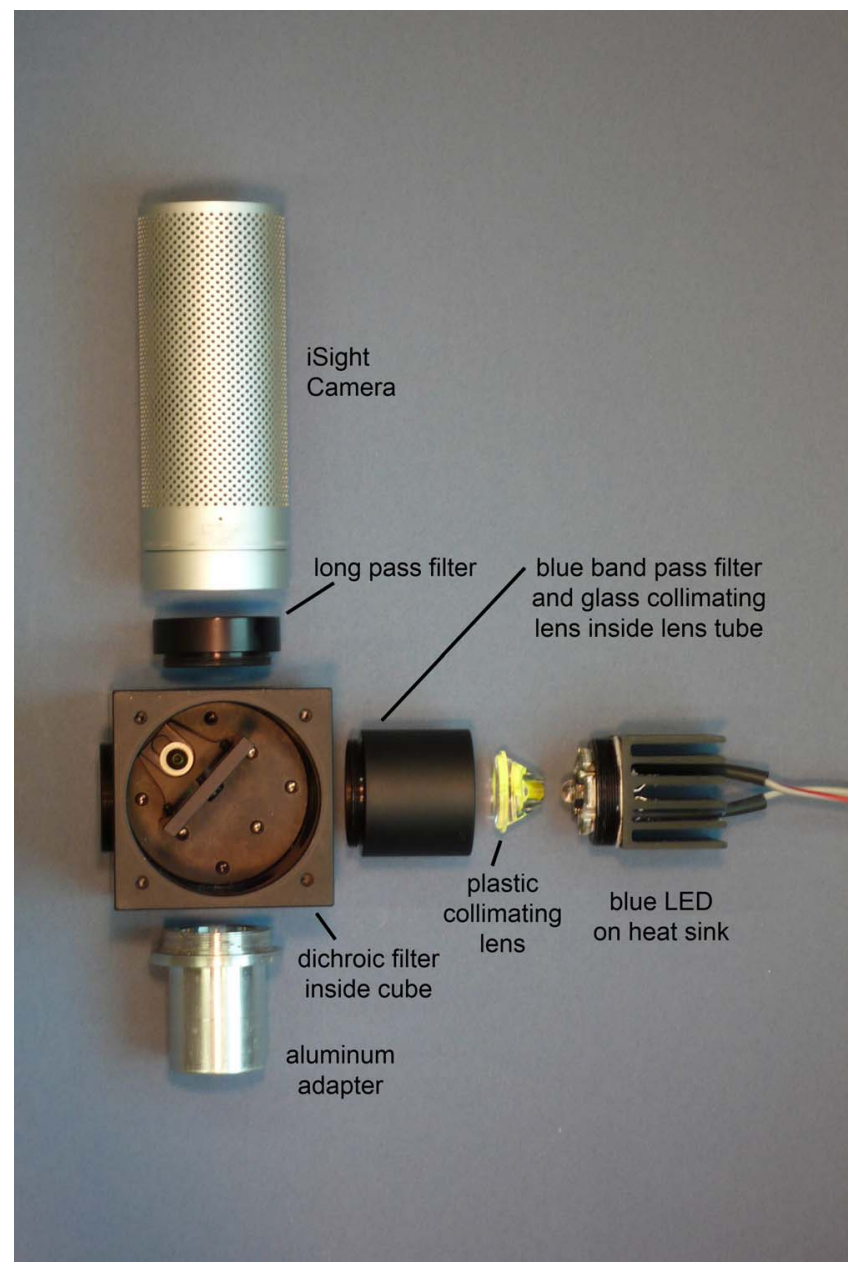

Fig. 2. (Color online) Exploded view of the fluorescence attachment.

\section{B. Fluorescence microscope}

Fluorescence microscopy is based on excitation of electronic transitions in fluorescent molecules within a sample and reradiation of their excitation energy via a two-photon process. Short wavelength light is used for illumination. Filters remove short wavelength light from the viewing path but pass the longer (visible) wavelength emitted by the sample. Fluorescent microspheres are available from suppliers in a variety of colors.

Converting the bright field microscope to view fluorescence requires three filters and an additional light source. Figure 2 shows an exploded view of the fluorescence attachment. A blue LED, in conjunction with collimating lenses and a blue filter, stimulates the fluorescence. The dichroic filter reflects the blue light to the sample and transmits the green light emitted by the fluorescent microspheres to the camera. A long-pass filter further attenuates unwanted reflections of the blue light. The blue filter sharpens the emission spectrum of the LED. Its tail would otherwise overlap the pass band of the long-pass filter. A cage cube mount holds the filters, the LED, and the camera in place. A green LED illuminates the sample from beneath. This is used as a focusing aid in bright field mode. Appendix $\mathrm{C}$ provides a component list of the fluorescence attachment.

Mounting the fluorescence attachment is not trivial. The optimal position of the CCD plane of the camera is $16 \mathrm{~cm}$ from the objective. However, the fluorescence attachment adds about $6 \mathrm{~cm}$ to the height of the camera. If the fluorescence attachment is mounted on top of the microscope eyepiece lens tube, the image quality deteriorates, although the magnification increases. The Spencer microscopes permit easy disassembly of the eyepiece lens tube, which fortuitously is $6 \mathrm{~cm}$ long and the optimal mounting scheme is to replace the eyepiece lens tube with the fluorescence attachment. The Bausch and Lomb microscopes can be permanently altered by shortening the eyepiece lens tube to accommodate the fluorescence attachment, as shown in Fig. 1.

\section{EXPERIMENTS}

\section{A. Brownian motion}

$1 \mu \mathrm{m}$ polystyrene microspheres are the standard particles used to observe Brownian motion. They are large enough to resolve in an optical microscope and small enough to remain suspended in a concavity slide for several hours. When suspended in distilled water, their sedimentation speed is of the order of $100 \mu \mathrm{m} / \mathrm{h}$. In order to give students the option of studying larger spheres, we used a mixture of glycerin and distilled water ${ }^{9}$ as the solute. The solute density matched the density of polystyrene, $1.055 \mathrm{~g} / \mathrm{cc}$, to about one part in 1000 . The density of solutions can be measured to this precision using low-cost hydrometers that can be obtained from suppliers of home wine and beer making materials. The solute density can also be tuned by using mixtures of deuterium oxide and distilled water.

Groups of students were given the task to observe Brownian motion of $1 \mu \mathrm{m}$ spheres and deduce the Boltzmann constant from their data. Each group made its own sample according to the procedure discussed in Appendix B 1. Before taking any quantitative data, students inspected their sample for convection, which is often caused by evaporation from the edges of the sample. They did this qualitatively by watching the microspheres for collective drift. If drift was observed, they made a new sample. A typical microscope image, obtained in this measurement, is shown in Fig. 3. When ready to collect data, the students used LOGGER PRO software to record time-lapse videos of the diffusing microspheres at intervals of $5 \mathrm{~s}$ over a $5 \mathrm{~min}$ period. A photograph of a stage micrometer was recorded on each workstation. Students calibrated the camera pixels to the micrometer's $10 \mu \mathrm{m}$ tick marks within LOGGER PRO. Axis directions were defined parallel to the edges of the CCD array. Viewing their time-lapse videos, students selected a single microsphere that stayed in the field of view for the full observation time. Clicking on it in each frame produced a data table of $x$ - and $y$-positions as a function of time. If it was not possible to observe a single microsphere for the desired length of time, students would choose a different microsphere and add its 


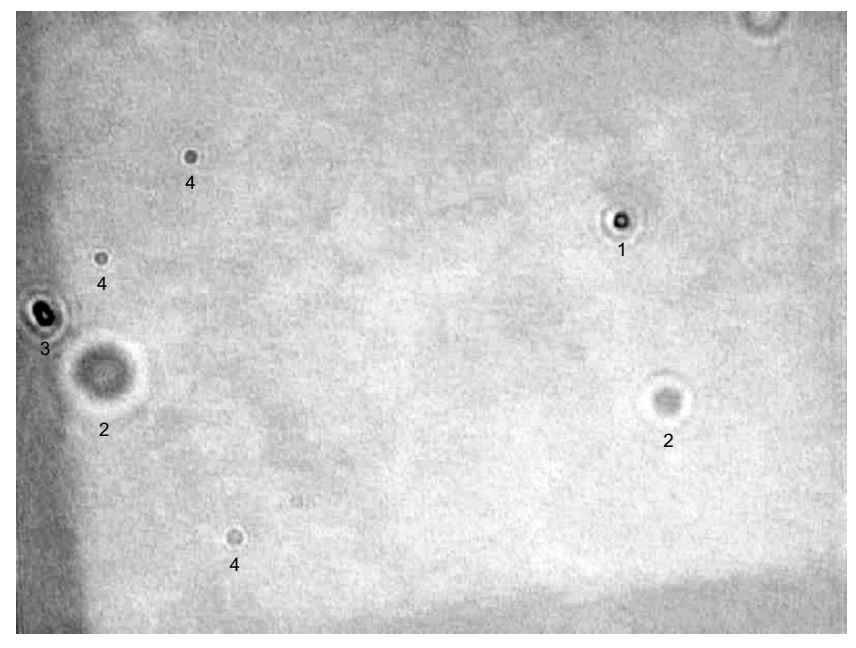

Fig. 3. A typical image of $1 \mu \mathrm{m}$ polystyrene spheres recorded by the iSight camera under blue (460-480 nm) illumination. Brightness and contrast were adjusted digitally. Feature no. 1 is a sphere in focus. Features no. 2 are spheres above or below the focal plane. Feature no. 3 is a pair of spheres stuck together and not fully resolved. Features no. 4 are dust particles on the CCD array.

displacements to their data table. Since the motion in the two dimensions is uncorrelated, each pair of images can be thought of as the end points of two one-dimensional diffusion measurements. Diffusion in the third dimension was not recorded. The same procedure was used for the data presented below, in this case for $2255 \mathrm{~s}$ intervals or a total of 450 displacements.

Einstein's analysis of Brownian motion was based on the molecular-kinetic theory of liquids and diffusion. Einstein concluded that the mean square displacement of particles suspended in a liquid is

$$
\left\langle x^{2}\right\rangle=2 D \tau,
$$

where $\tau$ is the observation time and $D$ is the diffusion constant. For spherical particles, $D=k_{B} T / 6 \pi \eta r$, where $k_{B}$ is the Boltzmann constant, $T$ is the absolute temperature, $\eta$ is the viscosity of the solution, and $r$ is the radius of the particle. For a set of displacements, we extract $\left\langle x^{2}\right\rangle$ and use Eq. (1) to calculate the diffusion constant and subsequently the Boltzmann constant.

Figure 4 shows histograms of displacements for 1,3 , and $10 \mu \mathrm{m}$ diameter microspheres suspended in an indexmatched water/glycerin mixture with viscosity of $(2.0 \pm 0.1) \times 10^{-3}$ Pas. In the absence of convection, the distributions are centered at zero and the widths squared are equal to the mean square displacement. Fit parameters are listed in Appendix D. The relative uncertainty in the mean square displacement is $\sqrt{2 /(N-1)}$, where $N$ is the number of observations. $^{10}$

The mean square displacement of the data set for $1 \mu \mathrm{m}$ diameter spheres is $2.4 \pm 0.2 \mu \mathrm{m}^{2}$ and the diffusion constant is $0.24 \pm 0.02 \mu \mathrm{m}^{2} / \mathrm{s}$, corresponding to $k_{B}=(1.5 \pm 0.1)$ $\times 10^{-23} \mathrm{~J} / \mathrm{K}$. The difference between the measured value of $k_{B}$ and the accepted value may be due to temperature varia-
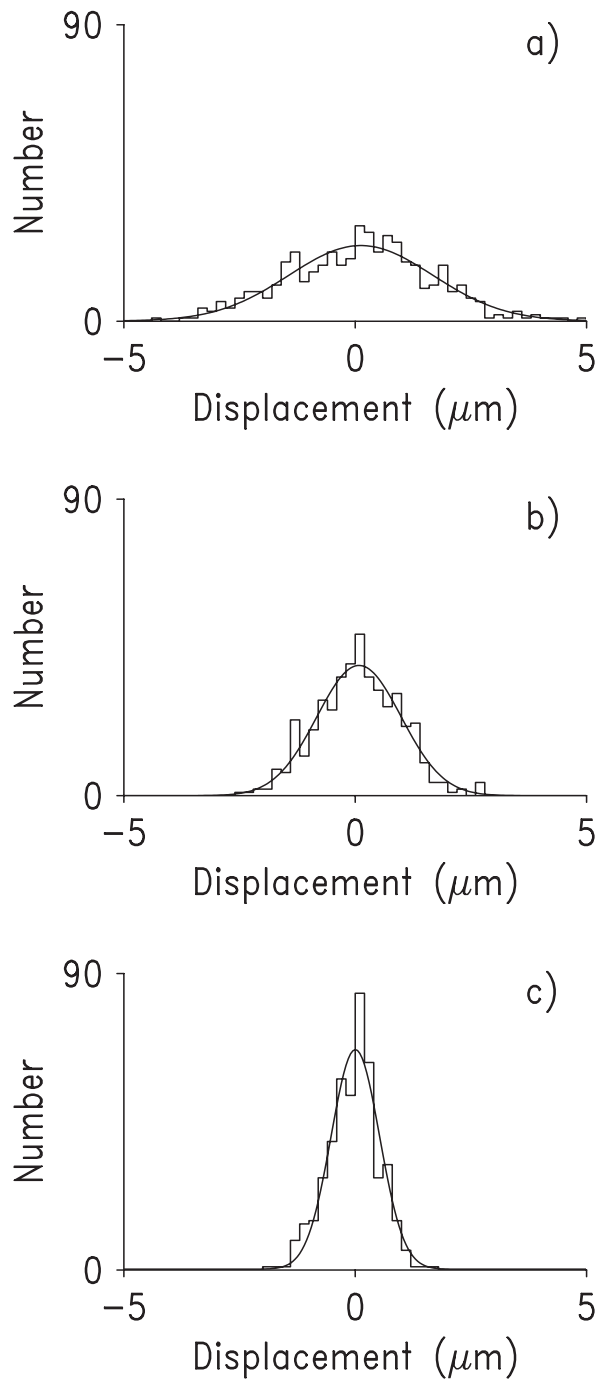

Fig. 4. Histogram of displacements for (a) $1 \mu \mathrm{m}$, (b) $3 \mu \mathrm{m}$, and (c) $10 \mu \mathrm{m}$ diameter polystyrene spheres suspended in a water/glycerin mixture with viscosity of $(2.0 \pm 0.1) \times 10^{-3} \mathrm{~Pa}$ s. Each data set represents 450 displacements. Gaussian fits are shown.

tions, which were not included in the analysis. The viscosity of the glycerin/water mixture changes by $5 \%$ per $2{ }^{\circ} \mathrm{C} .{ }^{11}$

The students also studied the diffusion of larger spheres. Figure 5 shows the diffusion constant as a function of sphere radius for the data shown in Fig. 4 plus an additional data set obtained with $6 \mu \mathrm{m}$ spheres. Fitting the data to an inverse- $r$ law yields a value for the diffusion constant $D$, which can be used to calculate $k_{B}=(1.6 \pm 0.1) \times 10^{-23} \mathrm{~J} / \mathrm{K}$.

\section{B. Properties of the fluorescence microscope}

To help students understand fluorescence microscopy, we ask them to disassemble the fluorescence attachment and measure the spectral properties of the components in a second laboratory session. Students wear disposable gloves to protect the optics from fingerprints. The Vernier/Ocean Optics "VIS-NIR Spectrometer" works nicely for the spectral measurements in conjunction with an incandescent light 


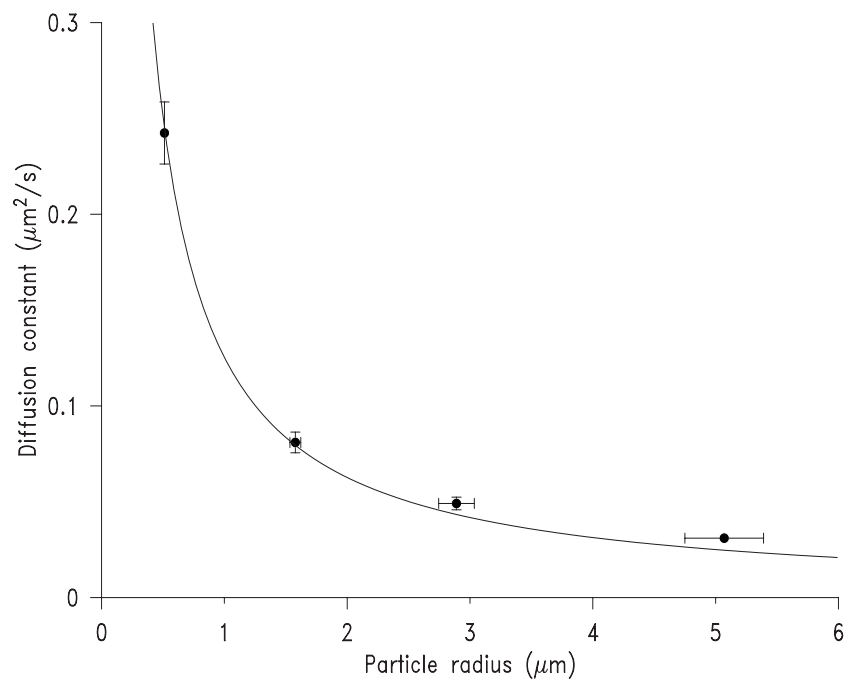

Fig. 5. Diffusion constant vs radius for polystyrene spheres suspended in a water/glycerin mixture with viscosity of $(2.0 \pm 0.1) \times 10^{-3} \mathrm{~Pa} \mathrm{~s}$. The solid line is the best fit to a $1 / r$ function.

bulb. A low-cost alternative is to use a photodiode to measure the filters' transmission of light with a multicolored set of LEDs.

The emission spectrum of the fluorescent microspheres was measured by placing a drop of the sphere solution on a white card and illuminating it directly with a blue LED. Each filter was measured separately and normalized to a white light spectrum. The spectra, multiplied by the appropriate filter response curves, give the normalized curves for illumination and emission shown in Fig. 6. The illumination distribution of the sample peaks at approximately $460 \mathrm{~nm}$; the filtered emission spectrum peaks at about $515 \mathrm{~nm}$. The difference between the peak position of the illumination spectrum and the peak position of the filtered emission spectrum is $55 \mathrm{~nm}$. This is the Stokes shift. The two curves were

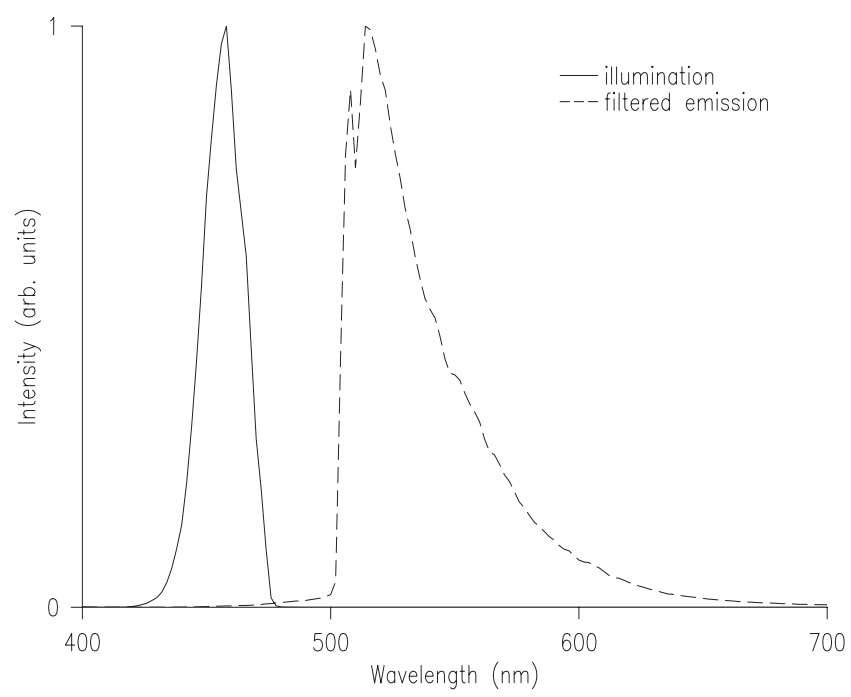

Fig. 6. Spectra of illumination and emission in the fluorescence microscope.
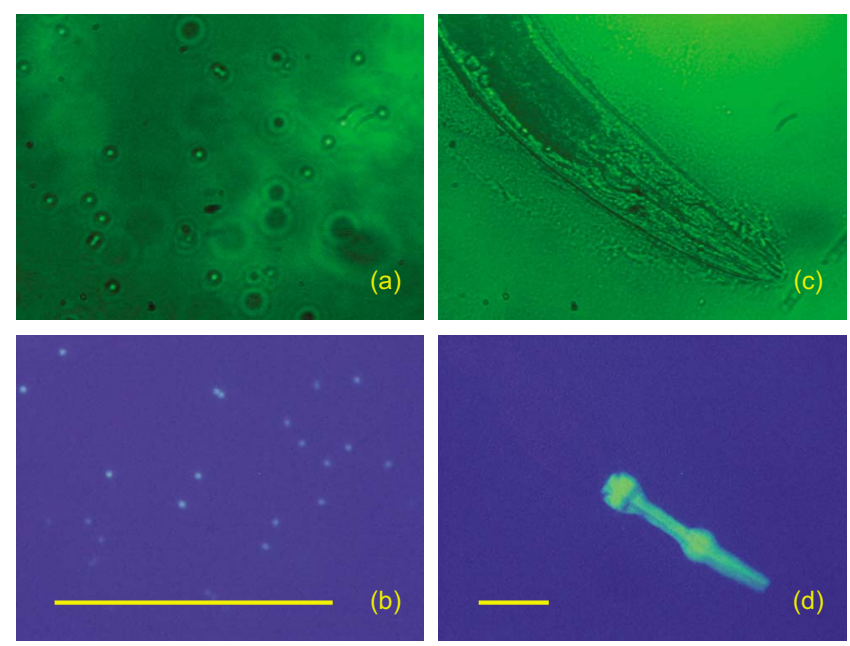

Fig. 7. (Color online) Sample images from the fluorescence microscope recorded with the Celestron NexImage USB camera. $1 \mu \mathrm{m}$ fluorescent polystyrene spheres viewed through a $40 \times$ objective in (a) transmission and (b) fluorescence. Adult transgenic $C$. elegans expressing green fluorescent protein (GFP) in pharyngeal muscle (strain CB5584) viewed through a $10 \times$ objective in (c) transmission and (d) fluorescence. The worms are mounted on $2 \%$ agarose pads containing $3 \mathrm{mM}$ sodium azide as anesthetic. The yellow bars represent lengths of $50 \mu \mathrm{m}$.

normalized separately. The illumination light is orders of magnitude brighter than the filtered light reaching the camera.

\section{Boltzmann distribution in a gravitational field}

Having understood how the fluorescence microscope works, students carry out a third laboratory exercise in which they measure the number density of fluorescent microspheres as a function of height in an equilibrium distribution and again derive the Boltzmann constant. Optionally, they can use the fluorescence attachment to view biological samples. Figure 7(a) shows a typical bright field image of $1 \mu \mathrm{m}$ fluo-

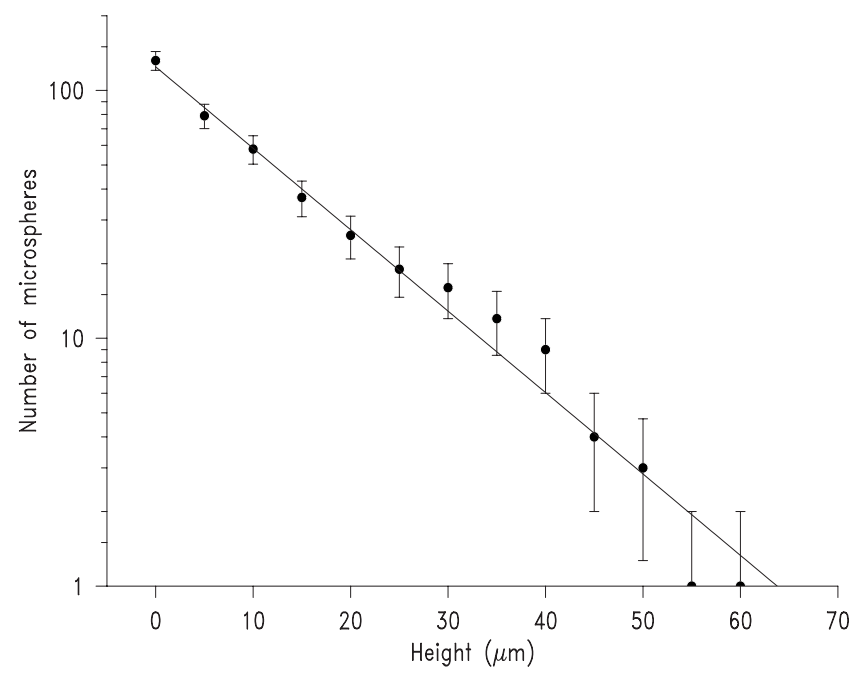

Fig. 8. Number of $1 \mu \mathrm{m}$ polystyrene spheres in the microscope focal plane as a function of height. The solid line is the best fit to an exponential function. 
Table I. A list of cameras suitable for microscopy applications. The packaging of each camera requires a unique adapter to mount it on the microscope. The Celestron camera comes with a short lens tube that fits snuggly over the lens tube of the Spencer.

\begin{tabular}{lcccccc}
\hline \hline Interface & Mfg. & Model & Pixels & $\begin{array}{c}\text { Max. frame rate } \\
\text { (frames/s) }\end{array}$ & $\begin{array}{c}\text { Price } \\
\text { (USD) }\end{array}$ \\
\hline USB & Celestron & NexImage & $640 \times 480$ & 30 & 153 & Celestron.com/neximage \\
USB & Point Grey & Chamelion & $1296 \times 964$ & 18 & 375 & Ptgrey.com/products/chameleon/index.asp \\
FireWire & Unibrain & Digital board camera 2040 & $640 \times 480$ & 30 & Unibrain.com/products/visionimg/fire_i_bc.htm \\
\hline \hline
\end{tabular}

rescent spheres with green $(510-540 \mathrm{~nm})$ illumination. Figure 7(b) was taken a few seconds later in fluorescence mode. Several spheres that are visible in Fig. 7(a) are out of the focal plane and are too dim to appear in Fig. 7(b). Images of a biological sample, C. elegans, are presented in Figs. 7(c) and $7(\mathrm{~d})$.

Several hours are required for a sample of $1 \mu \mathrm{m}$ spheres to settle at the bottom of a concavity slide. Accordingly, instructors prepared sealed samples at least 1 day before taking measurements using techniques described in Appendix B 2.

The focus controls of the microscope move the focal plane relative to the sample, parallel to the Earth's gravitational field lines. This direction defines the $z$-direction. To measure the distribution of microspheres, students photograph cross sections of the sample as a function of $z$ and count the microspheres that are in focus. Some of the Spencer micro- scopes were calibrated by the manufacturer. For uncalibrated microscopes, we measured the travel of the stage or lens tube with a dial caliper. Due to refraction at the air/sample interface, one must multiply the translation by the index of refraction of the solute to obtain the actual height from the apparent (measured) height.

The potential energy of a microsphere suspended in water is

$$
U(z)=\left(\rho_{\text {sphere }}-\rho_{\text {water }}\right) V_{\text {sphere }} g z .
$$

The number of spheres as a function of height follows the Boltzmann distribution,

$$
N(z)=N_{0} e^{-U(z) / k_{B} T} .
$$

Figure 8 shows a plot of the number of spheres as a function of $z$, with $z=0$ defined near the lower glass surface bounding

\begin{tabular}{|c|c|c|c|c|}
\hline Vendor & Part no. & Description & Qty. & $\begin{array}{c}\text { Price per } \\
\text { assembly } \\
\text { (USD) }\end{array}$ \\
\hline Thorlabs & $\mathrm{B} 1 \mathrm{C}$ & Blank cover plate & 1 & 14.00 \\
\hline Thorlabs & $\mathrm{B} 3 \mathrm{C}$ & Rotatable cover plate & 1 & 22.00 \\
\hline Thorlabs & B5C & 1 in. optic mount for $30 \mathrm{~mm}$ cube & 1 & 29.00 \\
\hline Thorlabs & $\mathrm{C} 4 \mathrm{~W}$ & Four-way mounting cage cube & 1 & 56.00 \\
\hline Thorlabs & ER3 & Cage assembly rod, 3 in. & 4 & 27.80 \\
\hline Thorlabs & SM1L10 & Lens tube, 1 in., 1 in. long & $1^{\mathrm{a}}$ & 15.00 \\
\hline Thorlabs & SM1L03 & Lens tube, 1 in., 0.3 in. long & 1 & 12.80 \\
\hline Thorlabs & SM1PL & SM1 plug & 2 & 30.00 \\
\hline Thorlabs & FD1 $Y^{\mathrm{a}}$ & Subtractive dichroic filter (yellow) & 1 & 21.60 \\
\hline Thorlabs & LB1437 & Collimating lens & 1 & 19.70 \\
\hline Fraen SRL & LXHL-NX05 & Acrylic lens $10\left(5^{\circ}\right.$ plastic collimating lens $)$ & 1 & 3.50 \\
\hline Digikey & HS-330-ND & BGA heat sink & 1 & 5.47 \\
\hline LuxeonStar & 5027-PB12 & Blue LED $470 \mathrm{~nm}, 700 \mathrm{~mA}$ & 1 & 8.54 \\
\hline LuxeonStar & 3023-D-E-700 & PWM 700 mA controller & 1 & 30.69 \\
\hline Mouser Electronics & $412-212104$ & $12 \mathrm{VDC}$ adapter, $1000 \mathrm{~mA}$ & 1 & 9.14 \\
\hline Chroma Technology & OS02333 & HQ455/50 blue band-pass filter & 1 & 40.00 \\
\hline \multirow[t]{2}{*}{ Chroma Technology } & OS02123 & E505LP long-pass filter & 1 & 35.00 \\
\hline & & Machined aluminum adapter ${ }^{c}$ & 1 & \\
\hline Duke Scientific & G0100 & Green fluorescent microspheres $(1 \mu)$ & $10 \mathrm{~mL}$ & 163.00 \\
\hline
\end{tabular}

Table II. Fluorescence attachment parts list.

${ }^{a}$ When reviewing manufacturer's specifications, recall that the spectral properties of the filter shift when light is incident at $45^{\circ}$.

bThese are "clearance filters" from Chroma Technology. The stock numbers referenced here are no longer valid, but comparable filters can be selected from overstock, subject to availability. A standard set of three filters, not from overstock, would cost $\$ 600$.

${ }^{c}$ The machined aluminum adapter has a 1.035 in., 40 tpi external thread on one end to match the mounting cube. The other end fits inside the microscope lens tube. A simple alternative is to use an additional Thorlabs SM1L10 to fit outside the microscope lens tube. This may require no machining, depending on the outside diameter of the microscope lens tube. 
Table III. The fit values of the amplitude $A$, the mean $\mu$, the standard deviation $\sigma$, and the constant $c$ with their uncertainties are listed.

\begin{tabular}{lcccc}
\hline \hline & $\begin{array}{c}A \\
\text { Data set }\end{array}$ & $\begin{array}{c}\mu \\
(\mu \mathrm{m})\end{array}$ & $\begin{array}{c}\sigma \\
(\mu \mathrm{m})\end{array}$ & $\begin{array}{c}c \\
(\text { counts })\end{array}$ \\
\hline Figure 4(a) $(1 \mu \mathrm{m})$ & $22.9 \pm 0.8$ & $0.122 \pm 0.050$ & $1.55 \pm 0.08$ & $0.1 \pm 0.6$ \\
Figure 4(b) $(3 \mu \mathrm{m})$ & $39.6 \pm 1.0$ & $0.082 \pm 0.026$ & $0.91 \pm 0.03$ & $0.0 \pm 0.4$ \\
Figure 4(c) $(10 \mu \mathrm{m})$ & $66.7 \pm 2.1$ & $0.002 \pm 0.018$ & $0.52 \pm 0.02$ & $0.3 \pm 0.6$ \\
\hline \hline
\end{tabular}

the sample. A numerical fit to determine the exponential height constant yields a Boltzmann constant of $k_{B}$ $=(1.4 \pm 0.1) \times 10^{-23} \mathrm{~J} / \mathrm{K}$.

\section{CONCLUSION}

The apparatus presented here will give a large number of students the opportunity to understand microscopy. The students can disassemble a fluorescence microscope and study its spectral properties. They can observe Brownian motion and the distribution of particles in sediment. These are experiments with important implications for biology and physics students. That one can use a microscope to observe, a camera to record, and a computer to track microscopic particles and thereby determine a number as miniscule as the Boltzmann constant is indeed impressive. These experiments give the students a hands-on feeling for statistics, a subject all too often treated as abstract mathematical manipulations.

\section{ACKNOWLEDGMENTS}

We gratefully acknowledge the many constructive comments on the manuscript received from the anonymous referees. We are likewise indebted to Dr. Chris Fang-Yen who prepared the samples of $C$. elegans and Steven Sansone of the Laboratory for Particle Physics and Cosmology machine shop, who machined the aluminum adapters for the bright field and fluorescence setups.

\section{APPENDIX A: INEXPENSIVE CAMERAS}

See Table I.

\section{APPENDIX B: SAMPLE PREPARATION TECHNIQUES}

\section{Basic sample preparation}

(1) Prepare a working solution by diluting $1 \%$ stock microsphere solution with distilled water or glycerin solution in a 1:1000 ratio for Brownian motion or a 1:200 ratio for the Boltzmann distribution. The volume of a single drop is about $40 \mu \mathrm{l}$.

(2) Rinse a concavity slide with ethanol and wipe.

(3) Fill the concavity with a few drops of room temperature solution. (Cold solutions release gas upon warming.)

(4) Place a cover slip on the slide and invert the slide on a paper wipe to blot the liquid.

(5) Rinse with water, if desired, to remove the glycerin residue.

(6) Dry with a wipe.
(7) (Optional) Apply a sealant with a syringe and dispensing needle or brush it on.

\section{Sealant}

(1) For short durations. Nail polish diluted in acetone. Sphere samples that have been sealed with nail polish clump and stick to glass surfaces within about 1 day. The presumed cause is that the solvent is miscible with water, thereby altering the surface properties of the spheres.

(2) For longer durations. Canada Balsam, diluted in xylene, or Permount (a toluene-based sealant, manufactured by Fisher Scientific). Both have been used to make samples that last for days to months.

\section{Density matching}

(1) Mixtures of glycerin:water of 1:3 work well. For $1 \mu \mathrm{m}$ spheres, there is very little, if any, clumping and sticking, and samples survive for at least a few days. The 3, 6, and $10 \mu \mathrm{m}$ samples clump and stick over time. For them, it was best to take data using new slides that had not settled out. It may help to add surfactant. (The sphere manufacturer recommends sodium dodecyl sulfate (SDS) in the range from $0.01 \%$ to $1 \%$.) We confirm the density match by monitoring the rise or fall in the particles for a few minutes.

(2) Spheres clump and stick immediately in saline solutions of the required density with or without surfactant added.

\section{APPENDIX C: FLUORESCENCE ATTACHMENT PARTS LIST}

See Table II.

\section{APPENDIX D: FIT PARAMETERS}

The data shown in Fig. 4 were fit with Gaussian functions,

$$
A \exp \left[-\frac{1}{2}\left(\frac{x-\mu}{\sigma}\right)^{2}\right]+c \text {. }
$$

(See Table III.)

\footnotetext{
${ }^{1}$ J. Perrin, Brownian Movement and Molecular Reality, translated by F. Soddy (Taylor \& Francis, London, 1910) [Ann. Chim. Phys. 18, 1-114 (1909)].

${ }^{2}$ Dongdong Jia, Jonathan Hamilton, Lenu M. Zaman, and Anura Goonewardene, "The time, size, viscosity, and temperature dependence of the Brownian motion of polystyrene microspheres," Am. J. Phys. 75, 111115 (2007).

${ }^{3}$ Ronald Newburgh, Joseph Peidle, and Wolfgang Rueckner, "Einstein, Perrin, and the reality of atoms: 1905 revisited," Am. J. Phys. 74, 478481 (2006).

${ }^{4}$ Paul Nakroshis, Mathew Amoroso, Jason Legere, and Christian Smith, "Measuring Boltzmann's constant using video microscopy of Brownian motion," Am. J. Phys. 71, 568-573 (2003).

${ }^{5}$ A. Einstein, Investigations on the Theory of the Brownian Movement, translated by A. D. Cowper (Dover, New York, 1956) [Ann. Phys. 322, 549-560 (1905)].

${ }^{6}$ F. Reif, Fundamentals of Statistical and Thermal Physics (McGraw-Hill, New York, 1965), pp. 201-211.

${ }^{7}$ For instructions on how to remove the lens from an iSight camera, see $<$ http://www.fas.harvard.edu/ scphys/isight/camera_mod.htm $>$.

${ }^{8}$ There are many inexpensive consumer-grade LED light sources such as the Sylvania "Dot-it Swivel" that can be used in place of a custom device with or without the microscope mirror.

${ }^{9}$ The density, viscosity, and index of refraction of glycerin/water mixtures
} 
at $20^{\circ} \mathrm{C}$ are tabulated in: CRC Handbook of Chemistry and Physics, 77th ed., edited by David R. Lide (CRC, Boca Raton, 1996), pp. 8-62, 63. The viscosity as a function of temperature is reported in: P. N. Shankar and M. Kumar, "Experimental determination of the kinematic viscosity of glycerol-water mixtures," Proc. R. Soc. London, Ser. A 444, 573-81
(1994).

${ }^{10}$ John R. Taylor, An Introduction to Error Analysis: The Study of Uncertainties in Physical Measurements, 2nd ed. (University Science Books, Sausalito, CA, 1997), Appendix E.

${ }^{11}$ See Ref. 9. Interpolation of temperature data in Table 1.

\title{
Tabletop demonstration of multiple source interference using ultrasonic transducers
}

\author{
E. J. Tucholski \\ Department of Physics, U. S. Naval Academy, Annapolis, Maryland 21402-5002
}

(Received 26 October 2007; accepted 14 June 2009)

\begin{abstract}
Superposition of multiple sources, constructive and destructive interference, and array beam forming are common topics demonstrated in undergraduate acoustic classes. The student learning experience is enhanced by building a system with speakers and measuring the resulting amplitude at various angles with a microphone. This experiment is often performed in an anechoic chamber to achieve quantitative agreement between theory and experiment. We have developed a simple tabletop apparatus using inexpensive ultrasonic transducers that yields comparable results without the need to use an anechoic chamber.
\end{abstract}

[DOI: 10.1119/1.3168976]

\section{INTRODUCTION}

Many acoustic classes use the rotating speaker array and microphone system described by Meiners ${ }^{1}$ to demonstrate the interference pattern of sound waves. This method provides a qualitative observation of the constructive and destructive interference directions. Challenges with this demonstration include the annoying loud tone and modal interference from objects in the room. To obtain results that agree quantitatively with theoretical predictions, the experiment is usually performed in an anechoic chamber.

The use of ultrasonic waves to demonstrate acoustic interference was proposed by Correll in the mid-1960s. ${ }^{2}$ Today, hobbyists, security systems, robotics, and other applications have developed a market for inexpensive ( $\sim \$ 5 /$ pair $)$ ultrasonic transducers, such as the ones shown in Fig. 1. These piezoelectric devices generate ultrasonic waves with wavelengths of roughly $1 \mathrm{~cm}$. These small wavelengths make it possible to create tabletop experiments that are more compact than their counterparts in the audible range and suggest a need to revisit and modify Meiners' original experiment.

The demonstration described here is simple and easy to perform on the tabletop of any physics laboratory or demonstration room. The apparatus scales with the ultrasonic wavelengths, much like commercially available microwave kits scale to demonstrate electromagnetic wave properties. ${ }^{3}$ The ultrasonic transducers are simple and inexpensive, yet robust enough to handle student misuse.

\section{MULTIPLE SOURCE INTERFERENCE}

The interference pattern from two or more omnidirectional acoustic sources in the far field is developed in standard acoustic texts. ${ }^{4}$ The phase difference $\phi$ between two sources, separated by a distance $d$, at some distant point at an angle $\theta$ from the line bisecting the sources is

$$
\phi \approx k d \sin \theta .
$$

The path difference traveled by the sound from the two sources to the far field is approximately $d \sin \theta$ and $k$ $=2 \pi / \lambda$ is the magnitude of the wave vector. The time averaged acoustic intensity (in units of $\mathrm{W} / \mathrm{m}^{2}$ ) in the far field is equal to

$$
\langle I\rangle=\frac{\left\langle\left(p_{1}+p_{2}\right)^{2}\right\rangle}{\rho v},
$$

where $p_{1}$ and $p_{2}$ are traveling pressure plane waves from the two sources in the far field, $\rho$ is the density of the medium, and $v$ is the speed of sound in the medium. The amplitudes

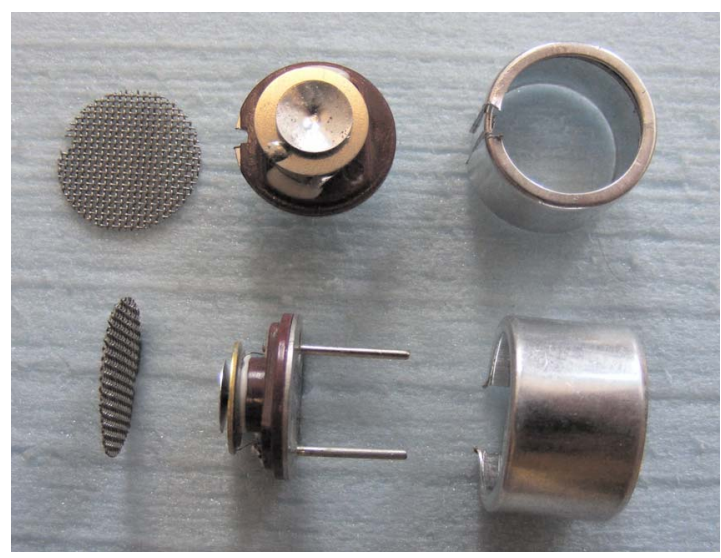

Fig. 1. Cutaway view of the piezoelectric ultrasonic transducers used in this work. 


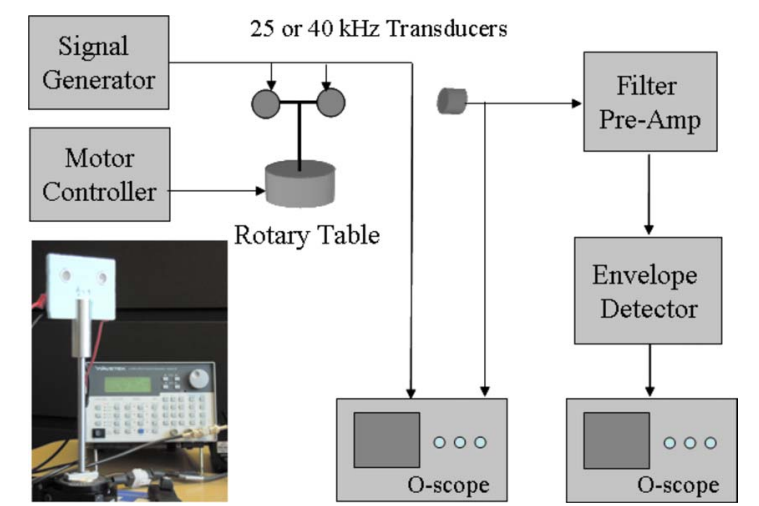

Fig. 2. A typical ultrasonic beamforming apparatus. The transmitting transducers are mounted on a rotary table and driven in parallel by a signal generator. The receiving transducer is mounted on a laboratory stand in the far field. One digital oscilloscope is used to monitor the amplitude of the receiver on a short time scale $(20 \mu \mathrm{s} /$ division $)$. The second digital oscilloscope views the envelope of the receiver signal on a long time scale (2 s/division) as the rotary table turns.

of $p_{1}$ and $p_{2}$ are acoustic pressures relative to the atmospheric pressure at the location of the receiver. The directional factor, also called the beam pattern function, that describes the normalized pressure field as function of the angle $\theta$ around the sources is defined as

$$
H(\theta) \equiv \sqrt{\frac{\langle I(\theta)\rangle}{\langle I(\theta=0)\rangle}} .
$$

The directional factor for two sources is

$$
H_{2}(\theta)=\left|\cos \left(\frac{\pi d \sin \theta}{\lambda}\right)\right| .
$$

The directional factor for an array of $N$ identical sources is

$$
H_{N}(\theta)=\left|\frac{1}{N} \frac{\sin \left(\frac{N \pi d \sin \theta}{\lambda}\right)}{\sin \left(\frac{\pi d \sin \theta}{\lambda}\right)}\right| .
$$

The angular locations of the maxima in the beam pattern for two sources [Eq. (4)], corresponding to constructive interference, require that

$$
d \sin \theta=m \lambda,
$$

where $m$ is any integer. The angular locations of the minima, corresponding to destructive interference, require that

$$
d \sin \theta=\left(m+\frac{1}{2}\right) \lambda .
$$

Equations (6) and (7) are studied when examining the interference pattern of two sources in a ripple tank or two-slit optical interference. $^{5}$

Equations (4) and (5) were developed to describe the beam pattern function around transmitting sources. The equations are equally valid to describe the beam pattern function around two passive omnidirectional receivers, connected in

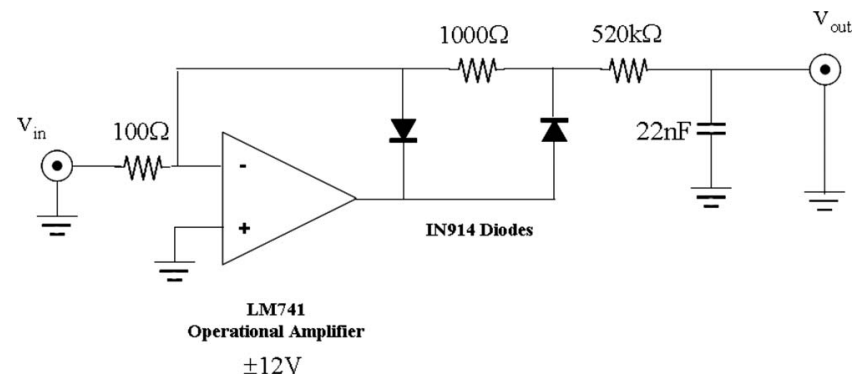

Fig. 3. The envelope detector circuit.

parallel, and placed on a rotating table with a single stationary transmitting source in the far field. The apparatus described in Sec. III can be modified to demonstrate either case.

\section{APPARATUS}

The essential elements of this demonstration are the small inexpensive ultrasonic ceramic transducers, shown in Fig. 1. These transducers utilize a piezoelectric ceramic disk bender design at a resonant frequency of either 25 or $40 \mathrm{kHz}$. The piezoelectric bender is bonded with a metal cone and mounted on a small can behind a protective screen with a diameter of $1.3 \mathrm{~cm}$. The diameters of the 25 and $40 \mathrm{kHz}$ transducer cones are 1.2 and $0.7 \mathrm{~cm}$, respectively. The nominal sound pressure level for the transmitter is $101 \mathrm{~dB}$ re $20 \mu \mathrm{Pa}$ at $1 \mathrm{~m}$ with a minimum receiver sensitivity of $-65 \mathrm{~dB}$ re $1 \mathrm{~V} / 0.1 \mu \mathrm{Pa},{ }^{6}$ both over a very narrow bandwidth around the resonant frequency. The transmitters are capable of dissipating over $10 \mathrm{~V}_{\mathrm{rms}}$. The receivers produce a $100 \mathrm{mV}$ signal when placed in the far field at a distance of $1 \mathrm{~m}$ from two or more transmitters. These piezoelectric transducers are typically sold in pairs, one optimized as a transmitter and the other optimized as a receiver. They are available from many suppliers. ${ }^{7,8}$

The ultrasonic transducers are installed in the configuration shown in Fig. 2, with two or more transmitters on the rotary table excited by a single signal generator. The motor controller turns the rotary table with a rotational period of about $30 \mathrm{~s}$. The rotary table is controlled with a joystick or with a computer using manufacturer supplied software or programs such as LABVIEW $\circledast$.

As the transmitting array turns, one oscilloscope displays the unprocessed waveform from the receiving transducer. The students observe constructive and destructive interference as relative maxima and minima in the waveform from the receiving transducer. An angular scale on the rotary table allows Eqs. (6) and (7) to be verified.

To obtain the directional factor around the rotating array, the output of the receiving transducer is sent to a filter preamplifier. The output impedance of the receiving transducer can be as high as $10000 \Omega$ near resonance and the input impedance of a Stanford Research Systems SR560 preamp- 

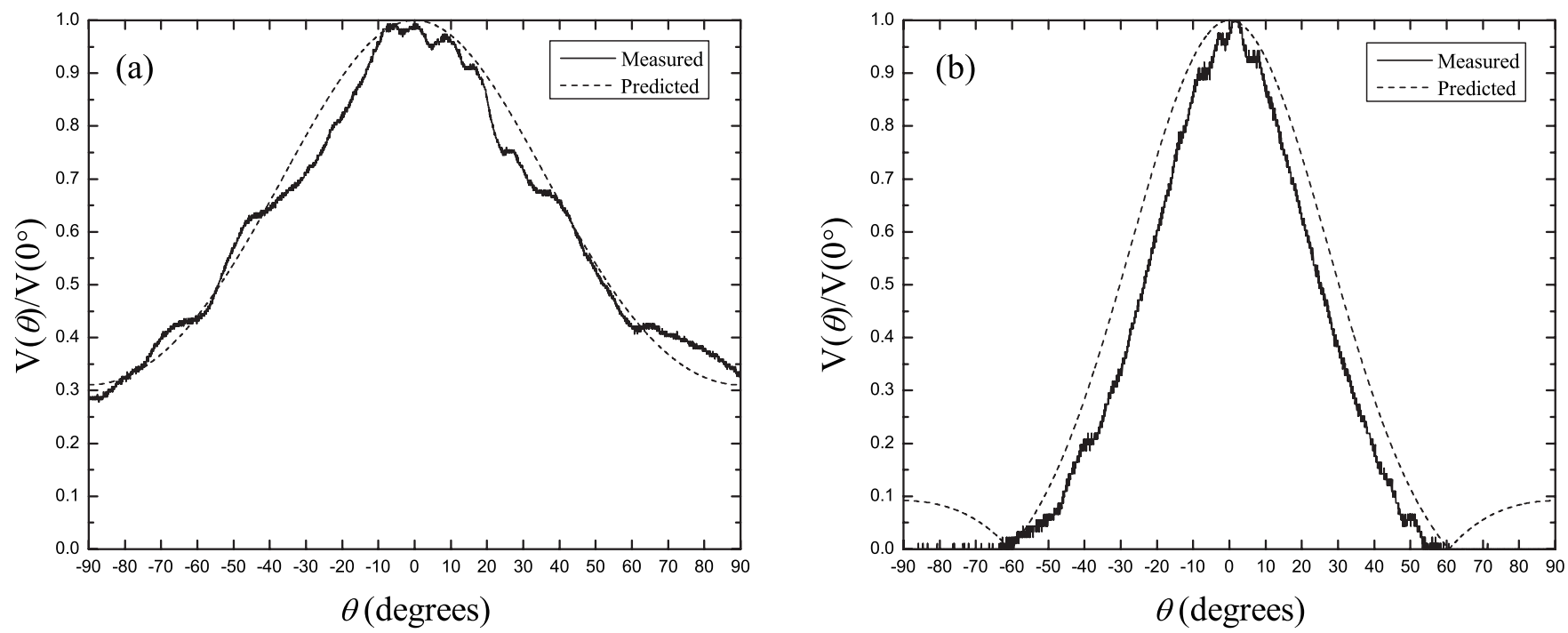

Fig. 4. The normalized directional factor for a single transducer transmitting at (a) $25 \mathrm{kHz}$ and (b) $40 \mathrm{kHz}$. A radius of $0.6 \mathrm{~cm}$ is used in the calculation of the directional factor of the baffled piston.

lifier is $100 \mathrm{M} \Omega$. After appropriate bandpass filtering (10$100 \mathrm{kHz}$ on the SR560), the signal leaves the low impedance $(50 \Omega)$ output of the preamplifier. An envelope detector, shown in Fig. 3, removes the 25 or $40 \mathrm{kHz}$ carrier and displays only the slowly varying peak signal. To function properly, the envelope detector requires an input of at least $1 \mathrm{~V}$, which can be obtained by selecting a gain of 20 on the SR560 preamplifier. While many oscilloscopes have a built-in peak detection function, the envelope detector completely eliminates the high-frequency carrier and displays only the upper envelope. The output of the envelope detector is sent to the second oscilloscope, set on a long time scale ( $\sim 2 \mathrm{~s} /$ division) to capture approximately $20 \mathrm{~s}$ of data as the rotary table turns. The captured trace is sent to a computer for analysis. It is convenient to collect the envelope over a range of $90^{\circ}$ on either side of the central maximum.

To map the beam pattern of a receiving array, simply swap the transmitting elements on the rotary table with receiving elements, place a transmitter in the far field where the receiver was previously, and swap the transmitting and receiving electronics. For more advanced demonstrations, the polarity of the leads for one of the transmitting elements can be swapped, placing the transmitters out of phase in a dipole fashion. ${ }^{9}$ This allows the demonstration of the beam pattern similar to Lloyd's mirror interference from a pressure release surface. ${ }^{10}$

Attention must be paid when mounting the transducers to prevent interaction between the elements. In our apparatus,
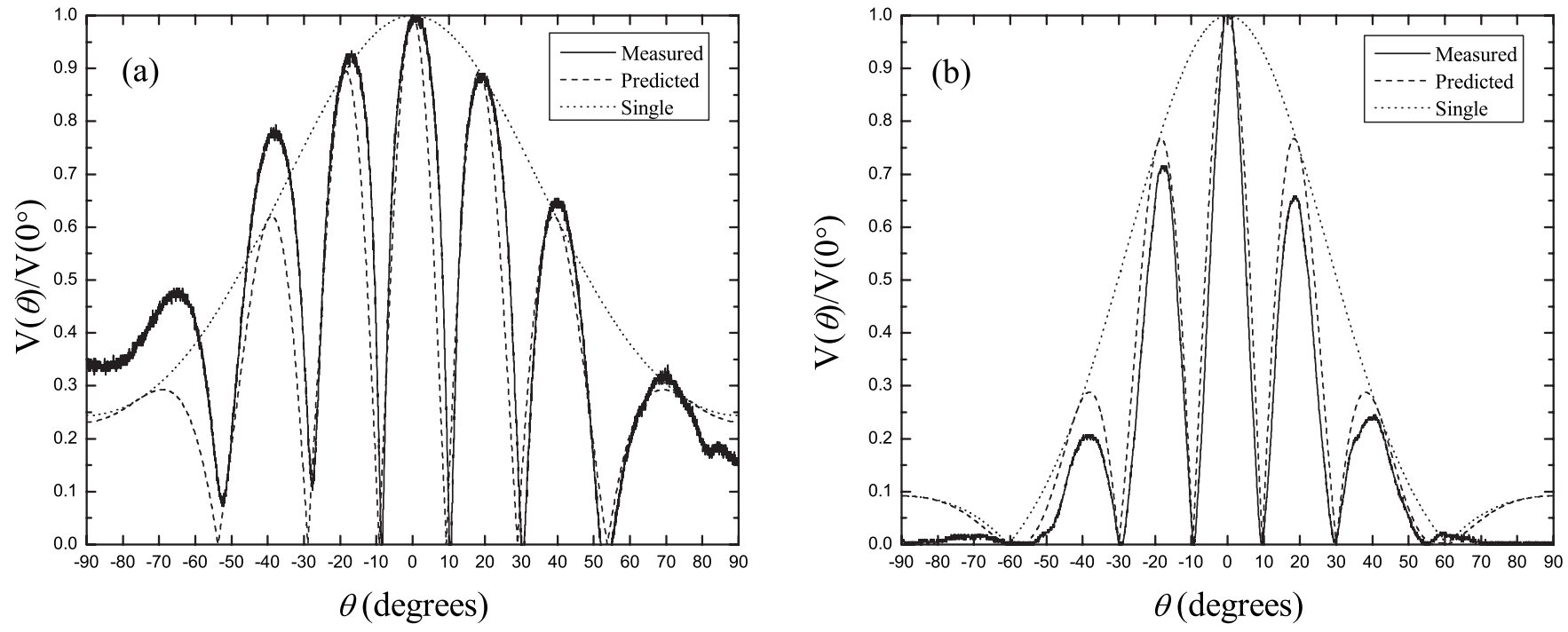

Fig. 5. The far-field beam pattern functions for (a) two $25 \mathrm{kHz}$ transmitters $(d / \lambda=3.1 \pm 0.1)$ and (b) two $40 \mathrm{kHz}$ transmitters $(d / \lambda=3.00 \pm 0.05)$. The validity of the product theorem is demonstrated by the single baffled circular piston source pattern, labeled "single," which bounds the two-source patterns. 


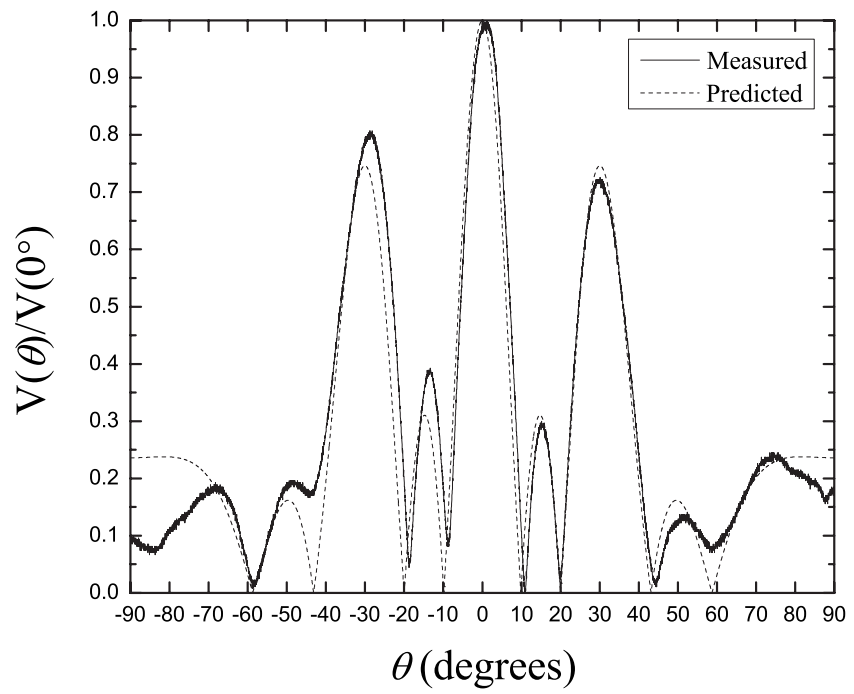

Fig. 6. The far-field beam pattern function for three $25 \mathrm{kHz}$ transmitters $(d / \lambda=1.95 \pm 0.05)$. As expected, one subsidiary maximum is clearly observed between adjacent major maxima.

the transducers were mounted on Styrofoam plates with holes cut to the outer diameter of the transducer can $(1.6 \mathrm{~cm})$. The distances between the centers of these holes were approximately integer multiples of the wavelength being used. Double sided foam tape secured the transducers in the plate. The temperature affects the speed of sound in air and requires the separation distance to wavelength ratio to be reevaluated. In practice, this ratio is used as a fit parameter.

In this apparatus, a single signal generator output was split and sent to both transducers. Occasionally, the voltage response of a transmitter varied from the nominal response, causing a distortion in the interference pattern. The effect is much like array shading where the amplitude of array elements is independently controlled. If multiple signal generators are available and can be synchronized in phase, each can be adjusted separately to obtain a balanced output. For advanced students, this offers the ability to demonstrate array shading and its effect on the beam pattern function. ${ }^{11}$

\section{RESULTS}

The transducers used have directional beam patterns and corrections to the omnidirectional source model, used to obtain Eqs. (4) and (5), must be applied. The design of these transducers results in a complicated geometry for the source of the ultrasonic wave. The bender component vibrates in a radial mode with a conical piston attached. Both components are placed in a cylindrical can with a slightly larger radius. Despite this complex construction, the source geometry of a single transducer can be approximated as a baffled circular piston with a directional factor given by

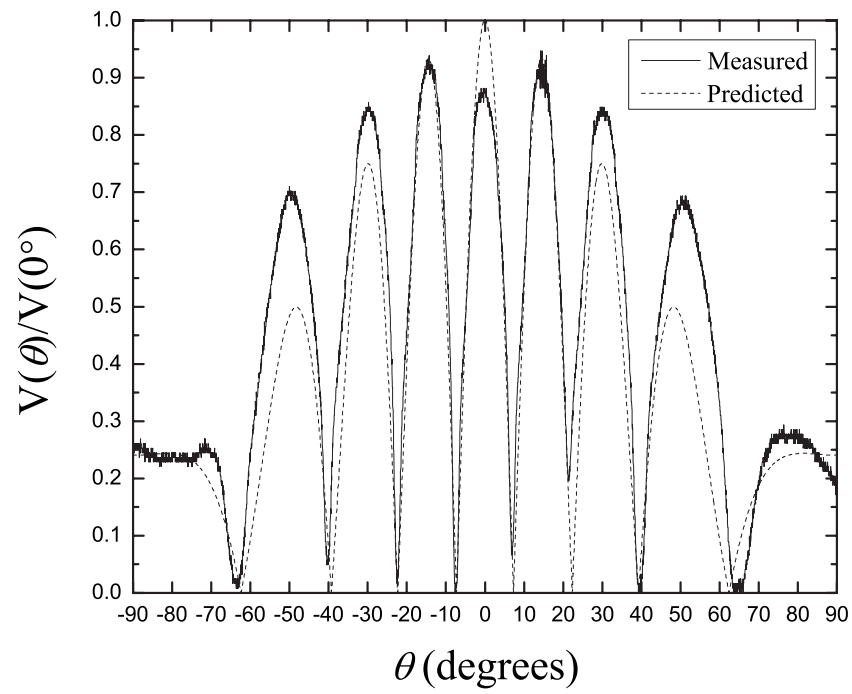

Fig. 7. Beam pattern function for two $25 \mathrm{kHz}$ receiving transducers $(d / \lambda$ $=3.95 \pm 0.05)$. A single transmitting transducer is placed in the far field.

$$
H_{\text {piston }}(\theta)=\left|\frac{2 J_{1}(k a \sin \theta)}{k a \sin \theta}\right|,
$$

where $a$ is the radius of the vibrating piston and $J_{1}$ is the first order Bessel function. Figure 4 shows the measured directional factors for a single transducer, compared to those predicted using the baffled piston approximation of Eq. (8) with a radius of $a=0.6 \mathrm{~cm}$. The good agreement allows the use of the product theorem to predict the interference beam pattern for two or more sources. The resulting direction factor is the product of Eq. (8) and either Eq. (4) or Eq. (5).

Typical results obtainable by students on a laboratory tabletop are shown in Figs. 5-7. Voltages obtained from the oscilloscope via the envelope detector are normalized and compared to the result predicted from the directional factor product theorem, shown as a dashed curve. While the side lobe amplitudes may vary by as much as $20 \%$ from the predicted amplitudes, the minima and maxima occur within $2^{\circ}$ of their predicted angular direction. In addition to transducer response variation already discussed, reflections from adjacent surfaces resulting in modal interference and alignment of transducers account for these differences. These results successfully demonstrate multiple source interference.

\section{ACKNOWLEDGMENTS}

The author is grateful to the Physics Department at the U.S. Naval Academy for supporting this course development and outfitting the Underwater Acoustics studio classroom with student laboratory stations to conduct this experiment. Professor Murray Korman designed the envelope detector for a related experiment and provided valuable discussions. Professor L. Tankersley and Professor C. Mungan lent their insights to improving the manuscript.

${ }^{1}$ H. F. Meiners, Physics Demonstration Experiments (Ronald, New York, 
1970), Vol. 1, pp. 508-509.

${ }^{2}$ M. Correll, "Ultrasonic transducers for interference experiments," Am. J. Phys. 32, iii-iii (1964).

${ }^{3}$ S. C. Bloch, "Electromagnetic and acoustic interference analog," Am. J. Phys. 33, 164-165 (1965).

${ }^{4}$ L. Kinsler, A. Frey, A. Coppins, and J. Sanders, Fundamentals of Acoustics, 4th ed. (Wiley, New York, 2000), pp. 195-199.

${ }^{5}$ P. Tipler, Physics, 2nd ed. (Worth, New York, 1982), pp. 448-451.

${ }^{6}$ For a discussion of decibels and acoustic source levels see D. Chapman and D. Ellis, "The illusive decibel, thoughts on sonars and marine mam- mals," Can. Acoust. 26 (2), 29-31 (1998).

${ }^{7}$ SensComp Global Components, "Application notes" <http:// www.senscomp.com/application_notes.html $>$.

${ }^{8}$ APC International Ltd. < http://www.americanpiezo.com/index.html $>$.

${ }^{9}$ H. Blum, "Acoustic interference demonstration," Am. J. Phys. 42, 413414 (1974).

${ }^{10}$ E. O. LaCasce and E. E. Hays, “An oceanographic Lloyd's mirror experiment,” Am. J. Phys. 52, 1121-1125 (1984).

${ }^{11}$ R. Urick, Principles of Underwater Sound, 3rd ed. (McGraw-Hill, New York, 1983), pp. 62-64.

\title{
Rotating crystal microwave Bragg diffraction apparatus
}

\author{
Joseph C. Amato ${ }^{\text {a) }}$ and Roger E. Williams ${ }^{\text {b) }}$ \\ Department of Physics and Astronomy, Colgate University, Hamilton, New York 13346
}

(Received 24 September 2008; accepted 9 June 2009)

\begin{abstract}
An easily constructed microwave Bragg diffraction apparatus is described, suitable for students in introductory physics classes. In contrast with previously published designs, only a single device, either the transmitter or the receiver, must be rotated in order to locate and unambiguously identify Bragg scattering maxima from several low-index $(h k l)$ crystal planes. Employing the "rotating crystal" technique, the orientation of the crystal need not be known a priori. This allows the students to use their measurements of $\theta_{\text {Bragg }}$ to determine the lattice spacing and the orientation of a concealed crystal, thereby mimicking the procedures used in real x-ray crystallography. (C) 2009 American Association of Physics Teachers.
\end{abstract}

[DOI: 10.1119/1.3160673]

\section{INTRODUCTION}

The microwave Bragg diffractometer is a familiar, timehonored experiment in the introductory physics laboratory. Previously published designs ${ }^{1-6}$ use a "crystal"- typically a cubic array of steel ball bearings or a square array of metal rods - that is visible to the students, and the lattice spacing and crystal axis orientation are thus known a priori. In addition, the transmitter and the detector must be rotated simultaneously by equal amounts relative to the crystal to ensure that the Bragg condition $\theta_{\text {inc }}=\theta_{\text {ref }}=\theta_{\text {Bragg }}$ is satisfied. In contrast, the apparatus described in this paper employs the "rotating crystal" technique, and only one device, either the transmitter or the detector, needs to be rotated by the students in order to find all Bragg reflections. Like the other designs, this apparatus is "two dimensional" in the sense that the transmitter, the receiver, and the crystal rotate in the horizontal plane about a common axis, centered on the crystal. In our setup, a motor spins the crystal rapidly, and all values of $\theta_{\text {inc }}$ are sampled during each revolution. The detector voltage is displayed on a storage oscilloscope, and the transmitter is slowly rotated to locate and maximize the Bragg reflections. (The rotating crystal technique is equivalent to powder diffractometry-albeit in two dimensions-for stationary samples.) Figure 1 shows that the scattering angles between the transmitter and the detector satisfy the following relations: $\theta_{\text {scatt }}=\theta_{\text {inc }}+\theta_{\text {ref }}$ and $\theta_{\text {inc }}=\theta_{\text {ref. }}$. The Bragg interference maxima are found when $\theta_{\text {scatt }}=2 \theta_{\text {Bragg. }}$. The Bragg angles depend on the wavelength $\lambda$ and the properties of the crystal,

$$
\frac{2 a}{\sqrt{h^{2}+k^{2}+l^{2}}} \sin \theta_{\text {Bragg }}=\lambda,
$$

where $a$ is the lattice spacing and $h, k$, and $l$ are the Miller indices identifying each family of crystal planes. For our two-dimensional geometry, $l$ is always zero. For the wavelength and the lattice spacing chosen here, the Bragg interference maxima from the (100), (110), (200), (210), and (120) crystal planes are easily found and identified.

The laboratory exercise has two parts. In the first part, the students use an exposed crystal to gain familiarity with the Bragg equation, learn the meaning of the Miller indices, and see directly the crystal planes associated with each set of indices. In the second part, students test their understanding of Bragg diffraction by confronting a concealed crystal, with the goal of determining its lattice constant and the orientation of its crystal axes. Because the Bragg angles are so quickly and easily found with this apparatus, both parts of the experiment can be completed successfully within a single $3 \mathrm{~h}$ lab period.

\section{APPARATUS DESCRIPTION}

The apparatus makes use of the familiar microwave transmitter and receiver modules found in the "microwave optics

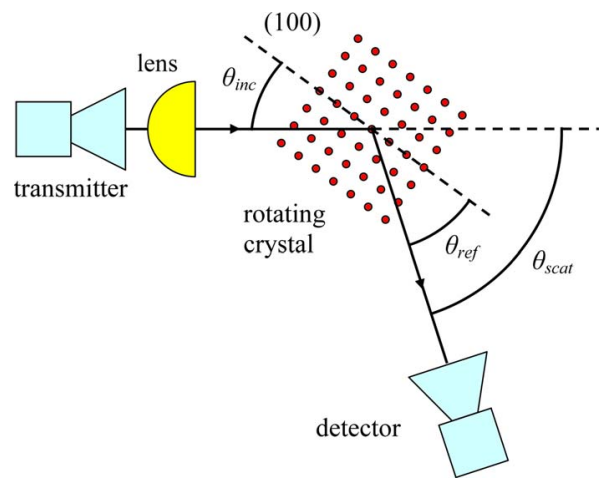

Fig. 1. Schematic view of the scattering geometry with all angles defined. 


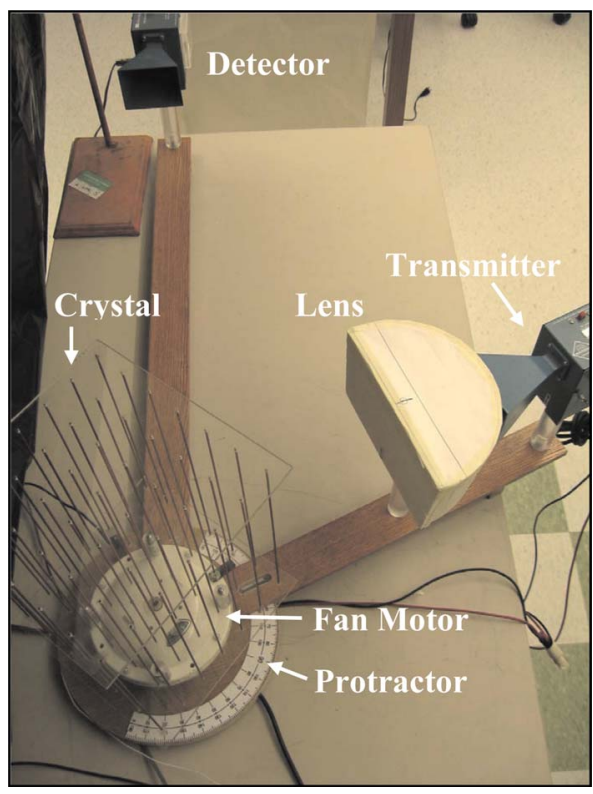

Fig. 2. Photograph of the apparatus. Note the slot in the transmitter arm for reading the protractor. A similar longitudinal slot, not visible in this photograph, allows adjustments of the lens position.

kit" marketed previously by Sargent-Welch and now by Pasco Scientific. The transmitter is a $10 \mathrm{~mW}$ Gunn diode oscillator emitting vertically polarized radiation at $10.525 \mathrm{GHz}(\lambda=2.85 \mathrm{~cm})$. The detector is a $1 \mathrm{~N} 21$ diode. The crystal is a square array of brazing rods, each having a diameter of $2.4 \mathrm{~mm}$ and a length of $30 \mathrm{~cm}$. The planar geometry of the apparatus allows us to simplify the structure by using long rods rather than spheres. ${ }^{6,7}$ The rods are sandwiched between two $6.4 \mathrm{~mm}$ polystyrene plates that are drilled to accept the rods, as shown in Fig. 2. The rigid assembly of plates and rods is mounted on an inverted ceiling fan motor that is rigidly attached to a circular plywood baseplate with a diameter of $30 \mathrm{~cm}$ and a thickness of $19 \mathrm{~mm}$. The fan motor is powered by either a variable transformer or a step down transformer with an ac output voltage of $24 \mathrm{~V}$. The rotation rate is approximately $170 \mathrm{rpm}$, close to $1^{\circ}$ per ms. The transmitter and detector are mounted on polystyrene posts with a diameter of $32 \mathrm{~mm}$, vertically centered on the crystal. The detector mounting post is screwed into a $1 \mathrm{~m}$ long wooden arm that is rigidly attached to the baseplate. The transmitter post is attached to a $60 \mathrm{~cm}$ long wooden arm that can rotate about the center of the baseplate. The scattering angle is measured using a simple protractor, made from a protractor image, expanded to match the baseplate diameter, printed on $8.5 \times 14$ in. label paper, and cut and glued to the perimeter of the baseplate. A slot cut in the transmitter arm allows $\theta_{\text {scatt }}$ to be determined with an accuracy of $\pm 1^{\circ}$

The transmitter is powered by an $8.5 \mathrm{~V}$ dc supply, which bypasses the disabled internal power supply within the transmitter module. The detector voltage is monitored with a dual-trace storage oscilloscope, displaying voltage vs time. A rotating flag, mounted on the fan motor housing, interrupts a photogate and is used to trigger the oscilloscope once per revolution. The $1 \mathrm{~N} 21$ detector voltage and photogate trigger pulse are displayed simultaneously on the scope. Strong sharply peaked wave forms with a signal-to-noise ratio greater than 20:1 are observed for reflections from the (100) and (110) planes when $\theta_{\text {scatt }}=2 \theta_{\text {Bragg. }}$.

Initially, the apparatus was plagued by the appearance of strong secondary peaks at angles $\theta_{\text {scatt }} \neq 2 \theta_{\text {Bragg. }}$. The more prominent of these, associated with the (100) planes, were comparable in magnitude to the "correct" peaks, which frustrated students' attempts to analyze and interpret their data. The extraneous peaks were eliminated by adding a crude collimating lens to the transmitter arm of the diffractometer. The lens is made from a semicircular Styrofoam trough with a diameter of $20.5 \mathrm{~cm}$ and a height of $12 \mathrm{~cm}$, packed with polyethylene beads. The lens can be slid along the transmitter arm to ensure that the transmitter is in the focal plane of the lens. The Styrofoam trough and beads were originally sold as part of the microwave optics kit offered by SargentWelch. Although they are no longer commercially available, the lens is quite easy to fabricate from scratch. Because of the long wavelengths, the required dimensional tolerances are modest. A variation in the focal length can be compensated by repositioning the lens relative to the transmitter. If the microwave modules are already on hand, the apparatus is simple and inexpensive to build. Detailed shop plans are available in the supplementary materials online. ${ }^{8}$ The diffractometer was displayed at the 2008 Summer Meeting of the AAPT in Edmonton, Canada.

\section{PROCEDURE}

The students begin the lab exercise by turning on the fan motor and obtaining a stable display on the oscilloscope triggered by the photogate voltage. They are advised to ignore observations for $\theta_{\text {scatt }}<20^{\circ}$, where the detected voltage arises mainly from radiation transmitted through the crystal. The students rotate the transmitter arm through increasing values of $\theta_{\text {scatt }}$ until strong reflections are detected. Figure 3(a) shows captured oscilloscope traces for Bragg scattering from the (100) crystal planes. The bottom trace in Fig. 3(a) shows the photogate voltage. The interval between the two photogate pulses covers one $360^{\circ}$ revolution of the crystal. The upper trace from the $1 \mathrm{~N} 21$ diode shows four equally spaced peaks per revolution, as expected from the fourfold symmetry of the crystal. A quick measurement of the time interval between reflections confirms that the crystal rotates $90^{\circ}$ between successive peaks. The transmitter arm is rocked back and forth to maximize the peak amplitude, and $\theta_{\text {scatt }}$ can typically be determined to within $1^{\circ}$ or $2^{\circ}$. At a larger $\theta_{\text {scatt }}$ the (110) Bragg peaks become prominent, as shown in Fig. 3(b). Like the (100) reflections, there are four equally spaced peaks per revolution of the crystal. The peaks shown in the upper trace in Fig. 3(b) have amplitudes similar to those of the (100) peaks, but they occur at distinctly different times, indicating that they arise from a different family of crystal planes. Figure 3(c) shows the eight peaks from the (210) and (120) planes, with a spacing of $40 \mathrm{~ms}$, or $37^{\circ}$, between the closest (120) and (210) planes. Figure 3(d) is a blowup of Fig. 3(b), showing that the detected peaks are symmetric with a FWHM of $5 \mathrm{~ms}$, or $4.6^{\circ}$, of crystal rotation.

Once a set of Bragg peaks has been located, the fan motor is turned off, and the crystal is brought to rest and slowly rotated by hand until the detector voltage is maximized. (Note that the oscilloscope trigger mode must be changed to "auto" to create a continuous display.) At this orientation of the crystal the Bragg condition $\theta_{\text {inc }}=\theta_{\text {ref }}=\theta_{\text {Bragg }}$ is satisfied. 

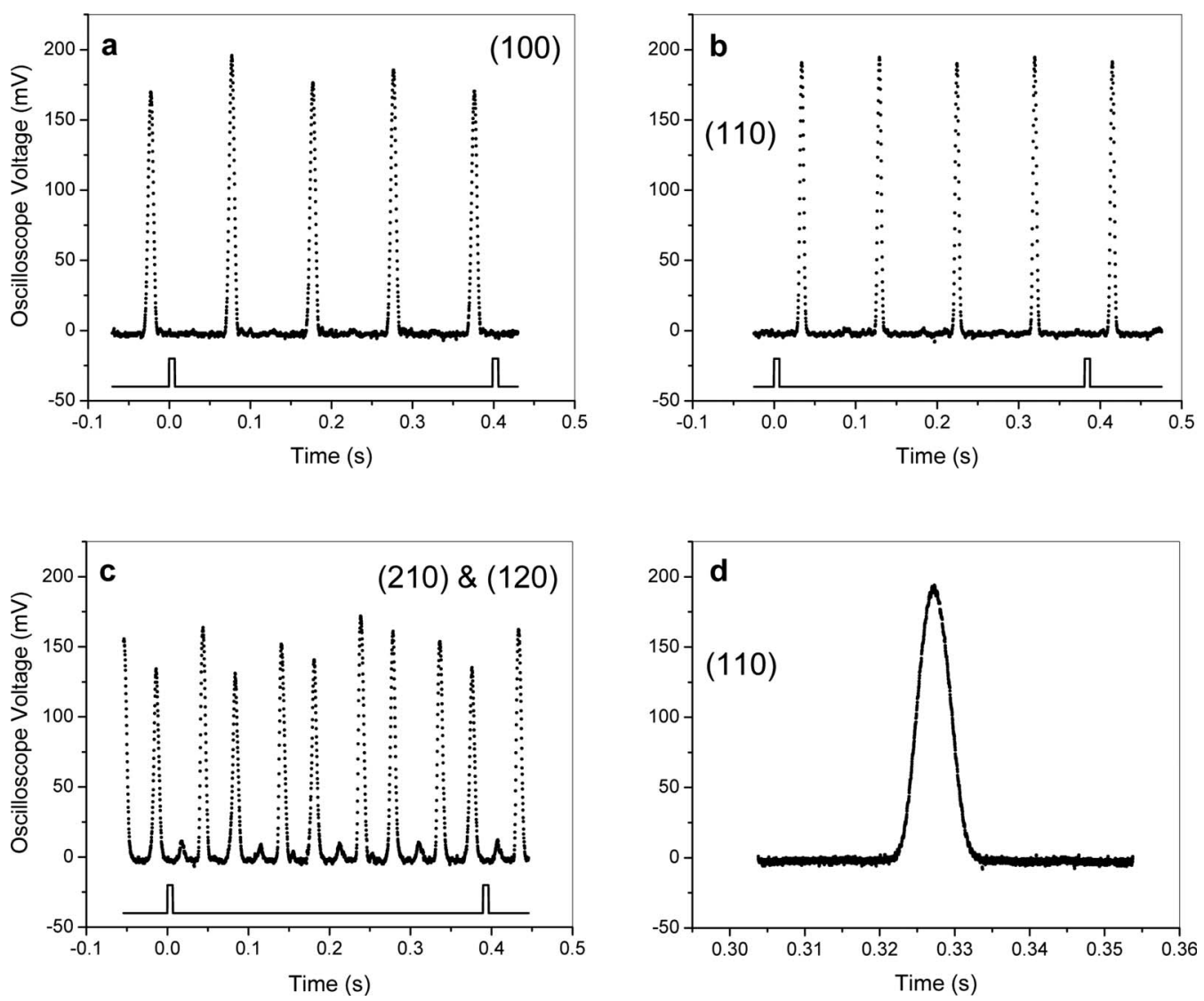

Fig. 3. (a) Oscilloscope traces for Bragg diffraction from the (100) planes. The top trace in the figure is the diode detector voltage. The bottom trace shows the photogate trigger pulses of magnitude $5 \mathrm{~V}$. (b) Same as (a) but for Bragg diffraction from the (110) planes. Note that the peaks appear midway between the peaks associated with diffraction from the (100) planes in (a). (c) Same as (a) but for Bragg diffraction from the (210) and (120) planes. (d) Expanded (110) peak, with a FWHM of $5 \mathrm{~ms}$, corresponding to a crystal rotation of $4.6^{\circ}$.

Students can easily see that a line drawn through the center of the crystal bisecting $\theta_{\text {scatt }}$ is parallel to a distinct family of crystal planes, and the planes responsible for a particular Bragg peak can be observed directly and identified unambiguously. This procedure is repeated for each set of Bragg peaks found with the rotating crystal. In addition to seeing the Bragg criterion satisfied directly, the students learn about

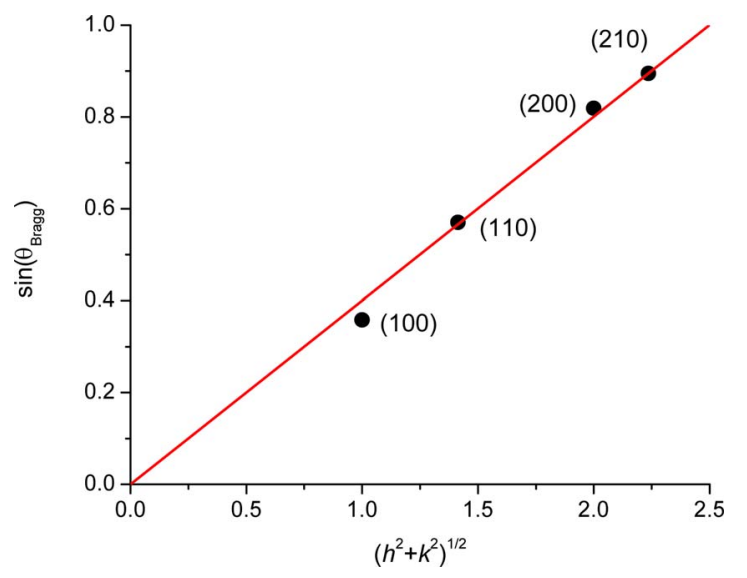

Fig. 4. The measured Bragg scattering angle as function of the Miller indices obtained with the exposed crystal. The slope of the linear fit is $\lambda / 2 a$ and is equal to $0.40 \pm 0.01$.
Miller indices in two dimensions and discover that, for example, a (200) reflection is merely a second order reflection from the (100) crystal planes.

\section{DATA AND ANALYSIS}

In our apparatus, we use a $7 \times 7$ square array of brazing rods with a lattice spacing of $3.7 \mathrm{~cm}$, and reflections from five families of planes are visible. The (120) and the (210) reflections are maximized at the same value of $\theta_{\text {scatt }}$, but they are spaced by $37^{\circ}$ rather than $90^{\circ}$, as can be verified easily by measuring the peak spacing on the oscilloscope display. Equation (1) shows that a plot of $\sin \theta_{\text {Bragg }}$ vs $\sqrt{h^{2}+k^{2}+l^{2}}$ produces a line with a slope of $\lambda / 2 a$. The measured slope yields a value of $a$ that can be compared to a direct measurement using calipers. For the data shown in Fig. 4, the best straight line fit to the data, forced to go through the origin, yields $a=3.56 \pm 0.09 \mathrm{~cm}$.

In the second part of the lab exercise, the exposed crystal is replaced by a concealed crystal, similarly constructed from brazing rods and polystyrene plates, with a lattice spacing of $3.2 \mathrm{~cm}$. In order to mask the orientation of the crystal, the polystyrene support plates are circular, and 61 rods are positioned in a square pattern, centered within a plate diameter of $29 \mathrm{~cm}$. The entire assembly is shrouded by an inverted plastic wastebasket. Because of the smaller lattice constant, only the (100) and (110) Bragg reflections are clearly visible; this 
was a deliberate design decision, meant to simplify the data analysis and ensure that the students can determine the crystal orientation and estimate $a$ successfully.

Our experience indicates that most beginning students can perform the lab exercise successfully without excessive supervision by the instructor. The entire exercise, including time for a short introductory discussion, data analysis, and report writing, can be completed easily within $3 \mathrm{~h}$. In the course associated with this exercise, Bragg diffraction and Miller indices are discussed within a single lecture. After completing the lab exercise, the students are able to respond correctly to homework and exam questions that address these topics.

\footnotetext{
${ }^{a)}$ Electronic mail: jamato@colgate.edu
}

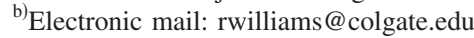

${ }^{1}$ Roland A. Allen, "Verification of Bragg's Law by the use of microwaves," Am. J. Phys. 23, 297-298 (1955).

${ }^{2}$ Robert G. Marcley, "Apparatus drawings project \#6: Bragg diffraction apparatus," Am. J. Phys. 28, 415-417 (1960).

${ }^{3}$ Thomas D. Rossing, Rodney Stadum, and Douglas Lang, "Bragg diffraction of microwaves," Am. J. Phys. 41, 129-130 (1973).

${ }^{4}$ T. G. Bullen, "An improved mounting for the Welch-Bragg diffraction apparatus," Am. J. Phys. 37, 333 (1969).

${ }^{5}$ William Henry Murray, "Microwave diffraction techniques from macroscopic crystal models," Am. J. Phys. 42, 137-140 (1974).

${ }^{6}$ M. T. Cornick and S. B. Field, "Microwave Bragg diffraction in a model crystal lattice for the undergraduate laboratory," Am. J. Phys. 72, 154158 (2004).

${ }^{7}$ Bailey L. Donnally, George Bradley, and Jacob Dewitt, "Models for microwave analogs of Bragg scattering," Am. J. Phys. 36, 920 (1968).

${ }^{8}$ See EPAPS Document No. E-AJPIAS-77-011909 for a complete set of shop drawings. This document can be reached via a direct link in the online article's HTML reference section or via the EPAPS homepage (http://www.aip.org/pubservs/epaps.html).

\title{
Construction and calibration of an impact hammer
}

\author{
Chris Waltham ${ }^{\text {a) }}$ and Andrzej Kotlicki \\ Department of Physics and Astronomy, University of British Columbia, Vancouver, British Columbia, \\ Canada V6T 1Z1
}

(Received 28 April 2008; accepted 23 June 2009)

\begin{abstract}
Impact hammers are used for rapid testing of the frequency-dependent response of structures to excitation. This work demonstrates how an impact hammer can be made from a piezoelectric crystal of the type widely used in barbeque lighters. The hammer was tested by measuring the inertance of a known mobile mass and by measuring the calculable driving-point admittance of rectangular wooden bars. The impact hammer was shown to be robust, easy to build, and to have a low-noise linear output for a wide range of applied forces. (C) 2009 American Association of Physics Teachers.
\end{abstract}

[DOI: $10.1119 / 1.3177062]$

\section{INTRODUCTION}

The principle behind the use of an impact hammer relies on the fact that a force of time duration $\Delta t$ contains Fourier components with all frequencies from zero to $1 / \Delta t$. Hence, a millisecond tap from an impact hammer excites the object being hit with all frequencies from 0 to $1000 \mathrm{~Hz}$. The response of the object can be measured with an accelerometer. If the applied force and acceleration are measured throughout the impact, the data can be used to determine the frequency response function ${ }^{1}$ (FRF) $H_{j k}$,

$$
H_{j k}(\omega)=\frac{X_{j}(\omega)}{F_{k}(\omega)},
$$

where $X_{j}$ is the displacement at position $j$ as a result of a force $F_{k}$ at position $k$. The FRF can be expressed in different ways, depending on whether the motion is described in terms of displacement, velocity, or acceleration. ${ }^{2}$ In this work, we will restrict ourselves to cases where $j=k$, normally referred to by the phrase "driving point." The "driving-point admittance" is the ratio of the velocity at a point to the force applied at that same point at a given frequency. Admittance has units of $\mathrm{s} / \mathrm{kg}$; impedance is its inverse and has units of $\mathrm{kg} / \mathrm{s}$. Inertance, or dynamic mass, is the ratio of force to acceleration and has units of $\mathrm{kg}$. All these quantities are complex, with an amplitude and phase.
Driving-point quantities can be used to find many mechanical properties of a structure and modal frequencies. A complete FRF, for many different values of $j$ and $k$, gives more extensive information, including modal shapes. In this work, we run the process in reverse and use the known properties of simple mechanical systems to calibrate and verify the behavior of a home-made impact hammer.

Impact hammers are widely available but are rather expensive, largely because they come calibrated. However, they are simple to build as piezoelectric crystals are cheap and easily obtainable; ours was obtained from an old barbeque lighter. Hence, with some care, a robust and reliable hammer can be fabricated for little cost using the procedures outlined below. Appropriate PC-based data acquisition systems are now also cheap, as are small accelerometers, due to their ubiquitous use as car airbag triggers. Once built, an impact hammer finds many uses, notably in lecture-demonstrations, where the speed of information gathering is impressive. Our hammer was specifically designed with musical acoustics in mind, being of appropriate size and sensitivity to make measurements on soundboards of instruments without causing any damage.

\section{THE IMPACT HAMMER SYSTEM}

\section{A. Piezoelectric crystals}

The behavior of piezoelectric crystals can be complicated. ${ }^{3}$ However, if one stays away from the reso- 

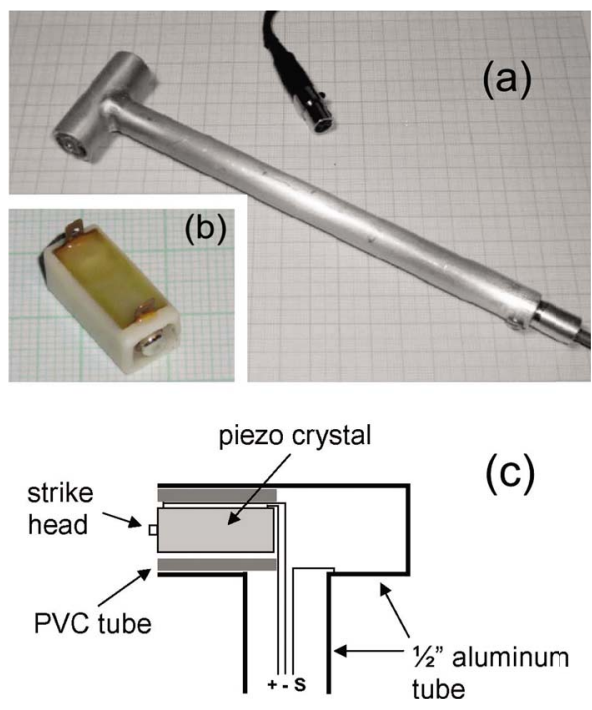

Fig. 1. (a) Impact hammer showing the aluminum tube construction and the mini-XLR connector. (b) Piezocrystal from a barbeque lighter with the striking mechanism removed. The background graph paper is marked with centimeter and millimeter grids. (c) Diagram of the arrangement of the piezocrystal and the electrical connections inside the hammer.

nance region, they can be modeled as a voltage source $V_{s}$ proportional to force $F$, in series with a capacitance with charge $q$. The measured voltage $V_{\text {meas }}$ across the input resistance of the data acquisition system is proportional to the current $d q / d t$, and the force is proportional to the time integral of $V_{\text {meas }}$. This relation is confirmed experimentally.

\section{B. Construction of the hammer}

In choosing a piezoelectric crystal for the hammer, we checked a selection of crystals from old barbeque lighters for signal size. A tap, light enough not to risk any damage to a musical instrument, easily produced a signal with an amplitude of several volts. The crystal was mounted inside a hammer, constructed from two 0.5 in. diameter aluminum tubes welded together, as shown in Fig. 1. The outputs from the crystal were connected to a three-wire cable with the third wire, the shielding wire, connected to the aluminum tube. A

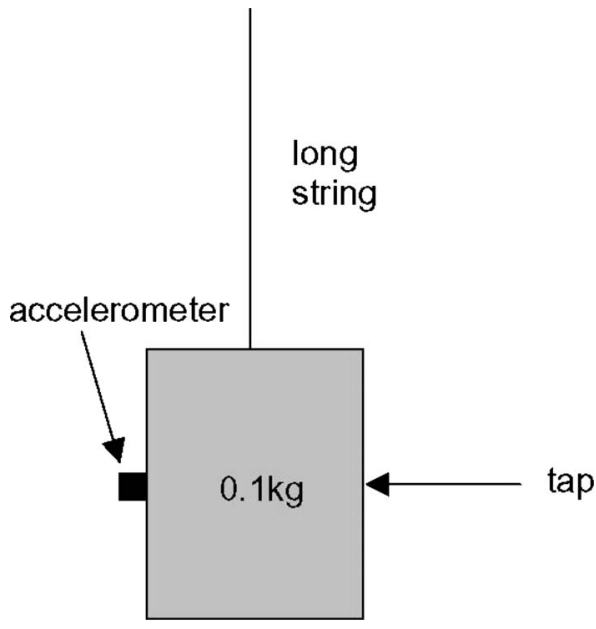

Fig. 2. Schematic of the configuration of the impact hammer and the accelerometer for the inertance test.
mini-XLR connector was attached to the other end of the cable. The continuous shielding between the piezoelectric crystal and the data acquisition system was crucial in reducing the noise to less than $1 \mathrm{mV}$. The hammer was held lightly at the end of the handle and swung against the system being tested such that it bounced once. The subsequent vibrations of the system were not affected by the mass of the hammer. The point of contact was the small metallic button on the end of the crystal, as shown in Fig. 1.

\section{Accelerometer and data acquisition}

To measure the acceleration of the system, a small and light $(0.2 \mathrm{~g})$ Endevco $25 \mathrm{~B}$ piezoaccelerometer ${ }^{4}$ was used. While not cheap, its tiny mass meant that only small corrections to the measured acceleration were required. Micromachined (MEMS) accelerometers could also be used; these were developed for airbag triggers and are inexpensive, with masses of a few grams or less.

The voltage outputs from the hammer and accelerometer were read by a Measurement Computing USB-1208FS analog-to-digital converter (ADC), ${ }^{6}$ mounted inside a metal box for shielding. The 12 bit ADCs operate at a maximum total sample rate of $50 \mathrm{kHz}$ (i.e., $25 \mathrm{kHz}$ for each of the two

(a)
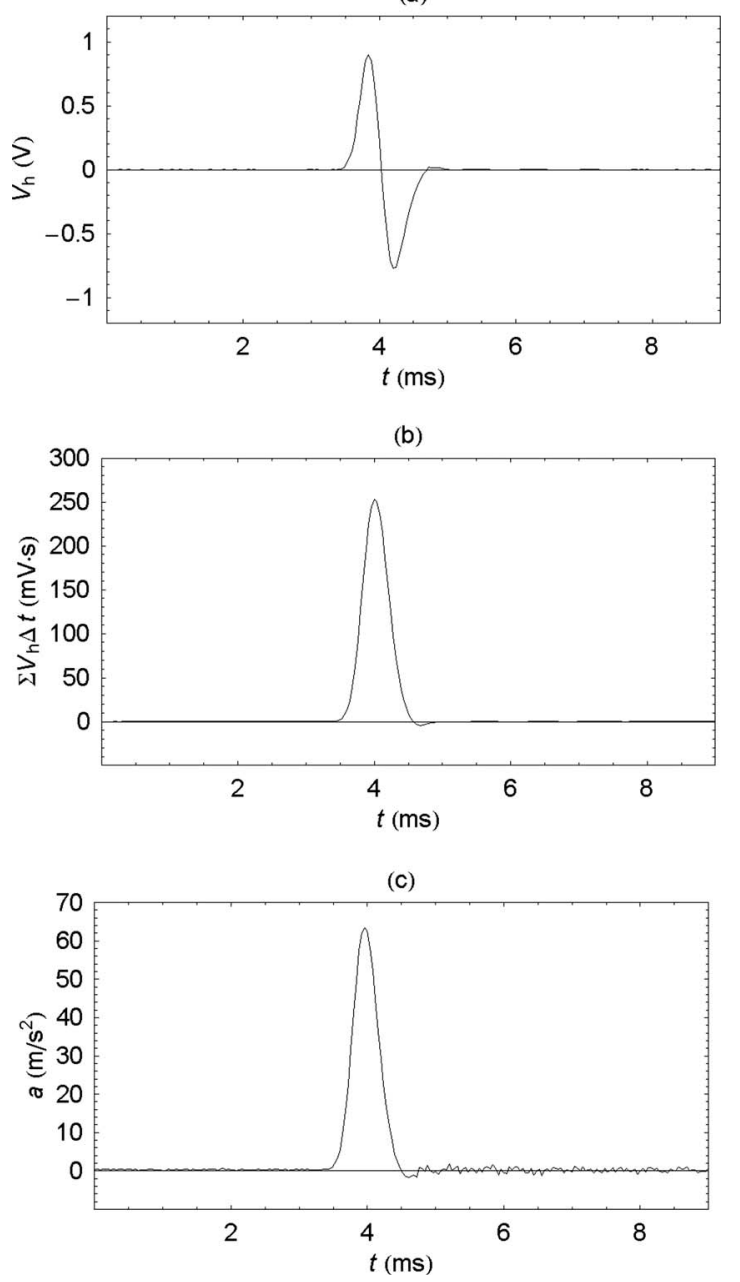

Fig. 3. (a) A typical impact hammer signal resulting from tapping a $100 \mathrm{~g}$ mass. (b) The time-integrated impact hammer signal. (c) The accelerometer signal resulting from a tap on the mass with the impact hammer. 

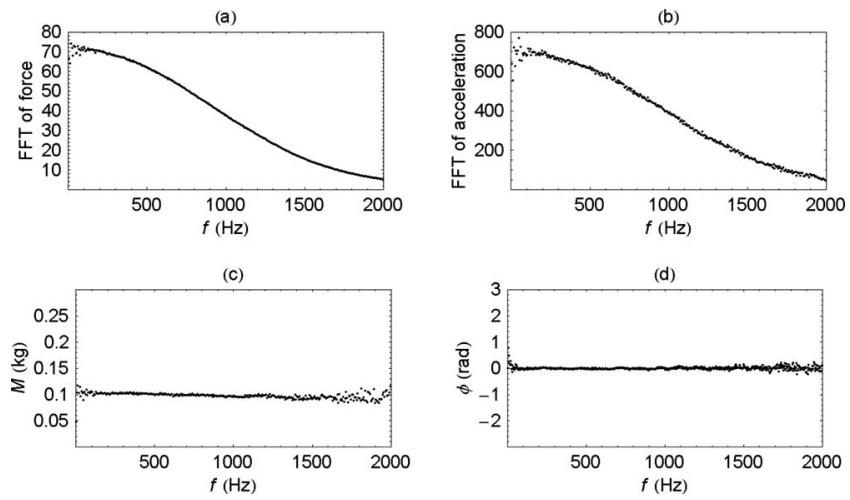

Fig. 4. (a) FFT of the integrated impact hammer signal. (b) FFT of the accelerometer signal resulting from a tap on the mass with the impact hammer. (c) Inertance of the $100 \mathrm{~g}$ mass. (d) Phase difference $\phi=\phi_{f}-\phi_{a}$ between the force on and the acceleration of the $100 \mathrm{~g}$ mass.

inputs used here). The ADC system was connected via USB to a standard laptop and operated by a visual basic data acquisition program.

There are two timing decisions to be made in making this type of measurement: how long to take data $\left(T_{s}\right)$ and at what sample rate $\left(f_{s}\right)$. The time $T_{s}$ depends on how long the response of the struck body lasts; $T_{s}$ should be long enough to encompass at least most of the signal but not too long as to include unnecessary noise. (It is possible to correct for the missing part of the exponential decay.) The minimum frequency resolution, $1 / T_{s}$, has to be small enough for the object being studied; objects with high quality-factors require better resolution. The sampling rate should be high enough to capture details of the fast hammer signal. In practice, a fast Fourier transform (FFT) size of 8192 is adequate and $T_{s}$ is set by the ADC sample rate. Windowing is not appropriate for the decaying signals.

\section{CALIBRATION TESTS}

\section{A. Inertance test}

The simplest way to calibrate an impact hammer is to tap an object of known mass that is completely free to move.

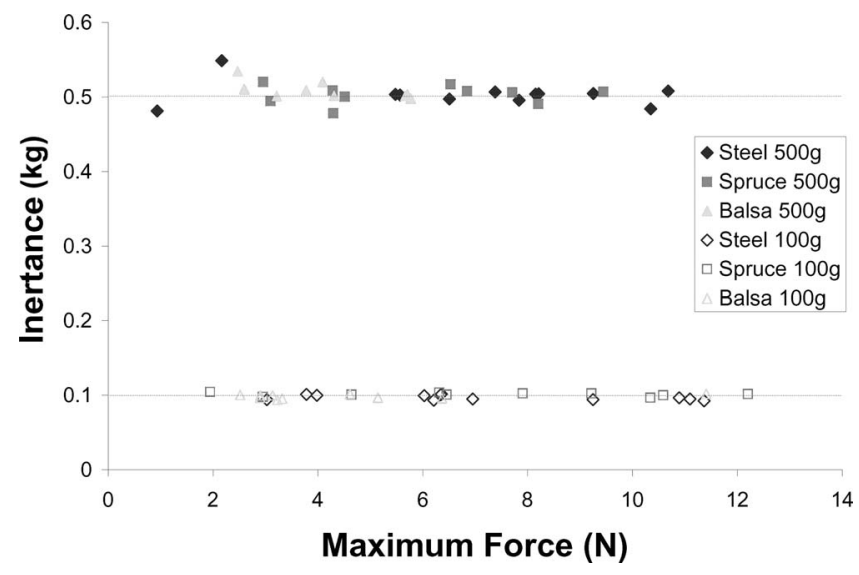

Fig. 5. Inertance of 100 and $500 \mathrm{~g}$ masses with various surfaces and measured with individual taps of different forces.
The ratio of the force and the acceleration is called the inertance. The inertance is real, independent of frequency, and equal to the mass of the object.

A test object was strung from a light $1 \mathrm{~m}$ long string, as shown in Fig. 2. The accelerometer was attached to one side with mounting wax at the same height as the center of mass. The mass was struck with the impact hammer on the opposite side. It was obvious when the strike was a good one, as the mass swung with no wobble. To test for any dependency of the results on the struck surface, small pieces of spruce and soft balsa were mounted on the object at the striking point.

The raw data from a tap are shown in Fig. 3. Figure 3(a) shows the hammer signal, Fig. 3(b) shows the time integral of the signal shown in Fig. 3(a), and Fig. 3(c) shows the response of the accelerometer. Figures 4(a) and 4(b) show the FFTs of the force and the acceleration signals, respectively. Figure 4(c) shows the inertance as a function of frequency, and Fig. 4(d) shows the phase between the force and the acceleration, which should be zero. The inertance and phase were used to calibrate the force signal. Figure 5 shows the results of tapping objects with different masses $(0.1$ and $0.5 \mathrm{~kg}$ ) and different surfaces (steel, spruce, and soft balsa). The useful range of frequencies is over $1 \mathrm{kHz}$ when tapping steel but no more than $400 \mathrm{~Hz}$ when striking balsa. The results were both linear in mass and constant for all surfaces and for a variety of maximum strike forces. The tap-to-tap standard deviation was 3.3\%. A small frequency dependence, corresponding to an $8 \%$ drop from 100 to $1000 \mathrm{~Hz}$, was reproducible and the calibration procedure thus used two constants: an overall frequency independent factor and a smaller term linear in frequency. The overall systematic error at a given frequency in this range was estimated to be $\pm 2 \%$. In order to produce a frequency independent phase, a correction was made for the $\sim 0.1 \mathrm{~ms}$ delay between the onset of the force and the acceleration pulses in the data stream.

\section{B. Admittance test}

The calibration procedure described in the previous section was verified with an admittance test. We measured the

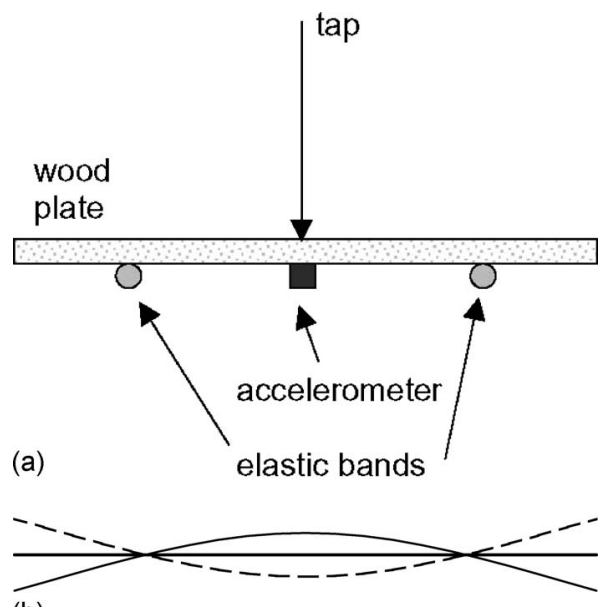

(b)

Fig. 6. (a) Schematic of the configuration of the impact hammer and the accelerometer for the admittance test. (b) The shape of the mode excited in the admittance test. The nodes are located $22.4 \%$ of the length of the bar from each end. 
central driving-point admittance of a free bar, a quantity that is calculable using independently measurable quantities, namely, the mass, the fundamental frequency, and the quality-factor. The bar was mounted on two pieces of thin elastic, positioned at the nodal points of the fundamental mode, $22.4 \%$ of the length from each end, as shown in Fig. 6 . The simple geometry allowed the central driving-point admittance $^{8} Y(x, \omega)$ to be calculated as function of position $x$, angular frequency $\omega$, and modal resonant frequencies $\omega_{n}$ :

$$
Y(x, \omega)=\frac{1}{M} \sum_{n=0}^{\infty} \frac{\psi_{n}^{2}(x)}{\omega_{n}^{2}-\omega^{2}} .
$$

$Y$ and $\omega_{n}$ in Eq. (2) are complex quantities. Equation (2) can be simplified in the region around the fundamental frequency $\omega_{0}$, provided other resonances are sufficiently far away,

$$
Y\left(x, \omega_{0}\right)=\frac{Q}{M \omega_{0}} \psi^{2}(x) ; \quad Q=\frac{\Re \omega_{0}}{2 \mathfrak{J} \omega_{0}} .
$$

In Eq. (3), $Y$ is expressed in terms of the quality-factor of the resonance $Q$, which is defined in terms of the real and imaginary parts of the complex fundamental frequency $\omega_{0}$, the mass of the bar $M$, and the modal shape of the fundamental resonance of the bar $\psi(x)$. The quality-factor was determined from the transient decay of the acceleration signal, which is a more sensitive method than using the width of the resonance. The fundamental angular frequency was obtained by fitting the peak of the admittance plot. The modal shape $\psi(x)$ is given by Eq. (4) (Ref. 9) and shown in Fig. 6,

$$
\begin{aligned}
\psi= & C\left(\cos \frac{k l}{2} \cosh k x+\cosh \frac{k l}{2} \cos k x+\sin \frac{k l}{2} \sinh k x\right. \\
& \left.+\sinh \frac{k l}{2} \sin k x\right) .
\end{aligned}
$$

The normalization factor $C$ in Eq. (4) is fixed by the requirements listed in Eq. (5),

$$
\int_{-l / 2}^{l / 2} \psi^{2}(x) d x=1 ; \quad \cos \frac{k l}{2} \cosh \frac{k l}{2}=1 .
$$

The bar was struck at its center, $x=0.5$, and for this vibration mode $\psi^{2}\left(\frac{1}{2}\right)=1.478$.

The first bar used was made of sitka spruce (Picea sitchensis, a few years old) with a mass of $44 \mathrm{~g}$, similar to that used in the musical instruments for which this impact hammer was created, being a part of a harp soundboard. The second bar was a heavier (301 g) piece of red oak (Quercus rubbra, at least 30 years old). In each case, the longitudinal direction of the grain ran along the length of the bar, and the
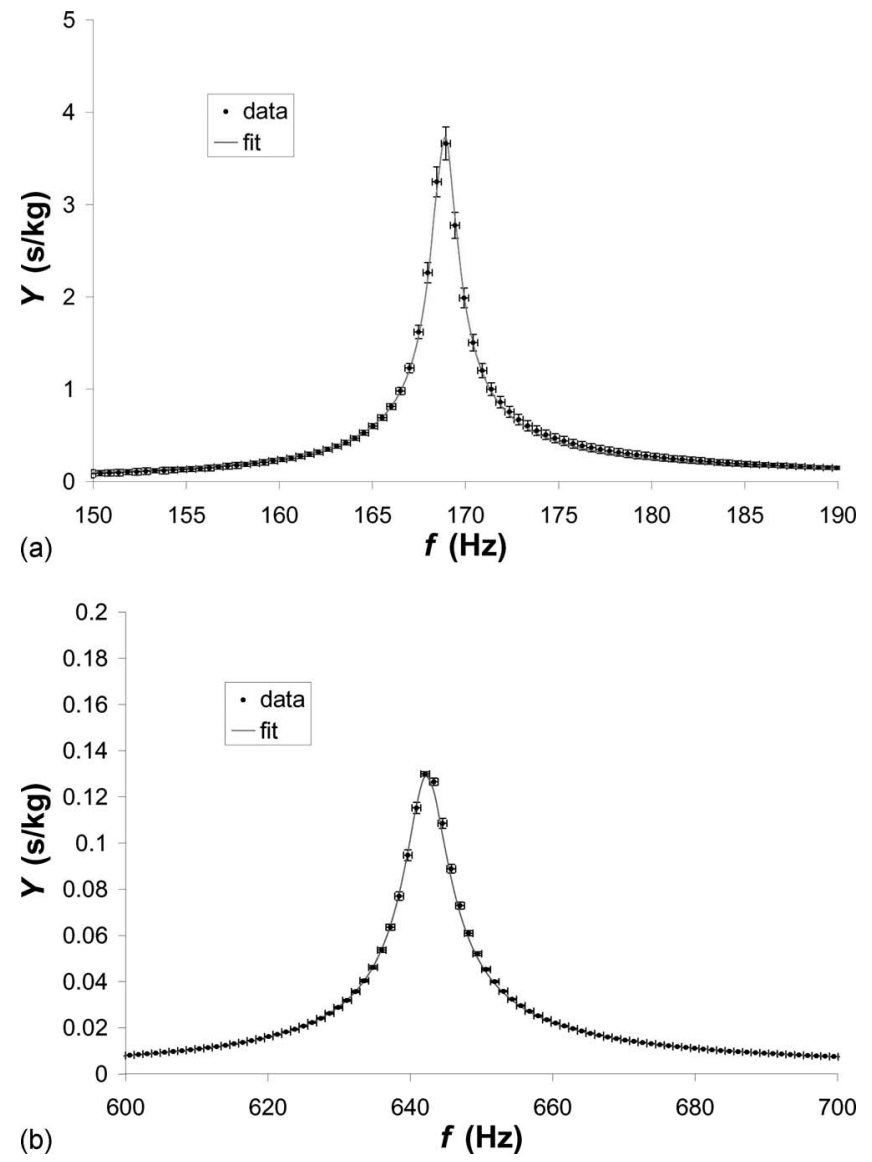

Fig. 7. Magnitude of the central driving-point admittance of free-free wooden bars: (a) Sitka spruce (44 g) and (b) red oak (301 g).

radial direction along its width. An FFT sample size of 8192 was used, with a sample rate of $4 \mathrm{kHz}$ for spruce and $10 \mathrm{kHz}$ for oak. This yielded a 2 and $0.8 \mathrm{~s}$ data sets, respectively, which captured essentially the entire response signal.

The theoretical admittance at the fundamental frequency was fitted to the form of Eq. (2). The fit included the third vibrational mode of the bar and the low frequency vibration of the supports $(\sim 15 \mathrm{~Hz})$, both of which had a small effect on the lineshape of the fundamental mode. The second vibrational mode has a node at the center of the bar and was not excited. The modal frequencies, the $Q$-factors, except for the fundamental $Q$-factor, which was fixed by the decay measurement, and the overall normalization factor were allowed to float. The mass of the accelerometer required a small correction to the resonant frequency $(0.3 \%)$ and the effective

Table I. Properties of wood samples used in admittance tests. The mass of the wood is the bare value; the accelerometer added $0.3 \mathrm{~g}$. The $Q$-factors were measured from the decay of the wood vibration; the linewidths apparent in the FFT lineshapes were a little larger due to frequency binning and the fitted $Q$-factors were thus somewhat smaller (128.0 for the spruce and 108.4 for the oak). The first error on $Y_{\text {fit }}$ arises largely from tap-to-tap variation in the admittance test; the second error is the $2 \%$ calibration error from the inertance test.

\begin{tabular}{lcccccc}
\hline \hline Wood & $\begin{array}{c}\text { Dimensions } \\
(\mathrm{mm})\end{array}$ & $\begin{array}{c}\text { Mass } \\
(\mathrm{g})\end{array}$ & $Q$ & $\begin{array}{c}f_{0} \\
(\mathrm{~Hz})\end{array}$ & $\begin{array}{c}Y_{\text {fit }} \\
(\mathrm{s} / \mathrm{kg})\end{array}$ & $\begin{array}{c}Y_{\text {thy }} \\
(\mathrm{s} / \mathrm{kg})\end{array}$ \\
\hline Spruce & $350 \times 72 \times 3.97$ & 44.0 & $130.0 \pm 0.5$ & $168.8 \pm 0.1$ & $3.74 \pm 0.19 \pm 0.08$ & 4.03 \\
Oak & $379 \times 52 \times 22$ & 301 & $109.0 \pm 0.2$ & $642.3 \pm 0.1$ & $0.129 \pm 0.002 \pm 0.002$ & 0.132 \\
\hline \hline
\end{tabular}


mass $(0.7 \%)$ of the spruce bar. The fits are plotted against the data in Fig. 7. Note that the horizontal "error bars" show the FFT bin-width. The vertical error bars were determined from the variation in the results of ten taps. A summary of fits and theoretical values is given in Table I. The theoretical values of the admittance at the peak of the resonance were calculated using the fitted values for $\omega_{0}$ and $Q$.

The response of a heavier oak bar was much easier to measure than the response of the lighter spruce bar; a larger fraction of taps produced good single pulses. (In principle a double-tap would be taken care of naturally by the FFT procedure, but in practice the hammer does not separate cleanly from the wood, preventing free vibration.) The tap-to-tap variation in the response of the oak bar was $6 \%$ around the resonance region, compared to $15 \%$ for the spruce bar.

The $Q$-factor for the oak bar was easier to extract from the time-decay of the response signal because the resonances were much more widely separated in frequency and the higher modes died away quickly, leaving a clean signal from the fundamental mode. The values found for the $Q$-factors were comparable with values given in the literature: 125 (Ref. 10) or 137 (Ref. 11) versus our 130 for sitka spruce and 90 (Ref. 10) versus our 109 for red oak (a 20\% difference between two samples not being exceptional).

A combination of all the above factors produced a value for the oak admittance with a quoted fractional error about three times as small as the fractional error for the spruce admittance. The measurements are more straightforward with a higher mass, and a higher well-separated frequency structure.

\section{CONCLUSION}

We have demonstrated how an impact hammer can be made from a piezoelectric crystal of the type widely used in barbeque lighters. With care in the choice of construction materials and cables, the device can produce a usable output with adequately low noise. The hammer was tested by measuring the inertance of a known mobile mass and the calculable driving-point admittance of a rectangular wooden bar. The hammer was shown to be robust, with a linear output over a wide range of forces and a small and reproducible frequency dependence.

\section{ACKNOWLEDGMENTS}

The authors thank Domenic diTomaso for technical assistance and also the referees of this journal for detailed and valuable comments.

\footnotetext{
${ }^{a)}$ Electronic mail: cew@phas.ubc.ca

${ }^{1}$ D. J. Ewins, "Modal analysis experimental: Basic principles," in Encyclopedia of Vibration, edited by S. Braun (Academic, San Diego, 2001), pp. 805-813.

${ }^{2}$ T. D. Rossing and N. H. Fletcher, Vibration and Sound (Springer, New York, 1995), pp. 117-122.

${ }^{3}$ P. M. Morse, Vibration and Sound (McGraw-Hill, New York, 1948), pp. $38-42$.

${ }^{4}$ Endevco Corporation 〈www.endevco.com〉.

${ }^{5}$ For example, the LIS3L02AQ three-axis accelerometer from STMicroelectronics $\langle w w w . s t . c o m\rangle$ is $3.4 \mathrm{~g}$ with a mounting board. The ADXL series from Analog Devices 〈www.analog.com〉 have a mass of about $0.1 \mathrm{~g}$. They come in leadless ceramic carrier packages but the wires can be hand-soldered directly on the package.

${ }^{6}$ Measurement Computing 〈www.measurementcomputing.com).

${ }^{7}$ J. M. M. Silva, "Modal analysis experimental: Measurement Techniques," in Encyclopedia of Vibration, edited by S. Braun (Academic, San Diego, 2001), pp. 813-820.

${ }^{8}$ Y. Chen, Vibrations: Theoretical Methods (Addison-Wesley, Reading, MA, 1966), pp. 208-211.

${ }^{9}$ T. D. Rossing and N. H. Fletcher, Vibration and Sound (Springer, New York, 1995), pp. 57-60.

${ }^{10}$ I. Barducci and G. Pasqualini, "Measurement of the internal friction and the elastic constants of wood," Nuovo Cimento 5, 416-466 (1948).

${ }^{11} \mathrm{~V}$. Bocur, Acoustics of Wood (Springer, Berlin, 2006), p. 178.
}

\title{
Measurement of electrical conductivity in nonferromagnetic tubes and rods at low frequencies
}

\author{
J. Íñiguez, V. Raposo, and M. Zazo \\ Departamento de Física Aplicada, Universidad de Salamanca, E-37071 Salamanca, Spain
}

(Received 27 February 2008; accepted 1 July 2009)

\begin{abstract}
We present a simple and precise method to measure the azimuthal electrical conductivity in nonferromagnetic tubes and rods at low frequencies. The results are readily obtained by analyzing the eddy currents in the sample when it is subjected to an axial harmonic magnetic field. The information about the eddy currents can be obtained from a measurement of the phase of the induced voltage in a short probe coil located inside the tube or around the rod when the sample is axially excited by a long primary coil. At low frequencies, the eddy currents are distributed over the full depth of the sample and any irregularities can be detected when the probe coil is moved along the sample axis. (C) 2009 American Association of Physics Teachers.
\end{abstract}

[DOI: $10.1119 / 1.3184154]$

\section{INTRODUCTION}

The determination of electrical resistivity in metals by inductive methods is a good alternative to conventional fourprobe techniques. To achieve accurate results, it is essential to have a very precise knowledge on the magnetic permeabil- ity of the material and to operate with simple geometries. The first requirement poses a serious problem when studying ferromagnetic metals since the magnetic permeability is usually not known with sufficient precision. Therefore, in our application, we study only nonferromagnetic materials with 


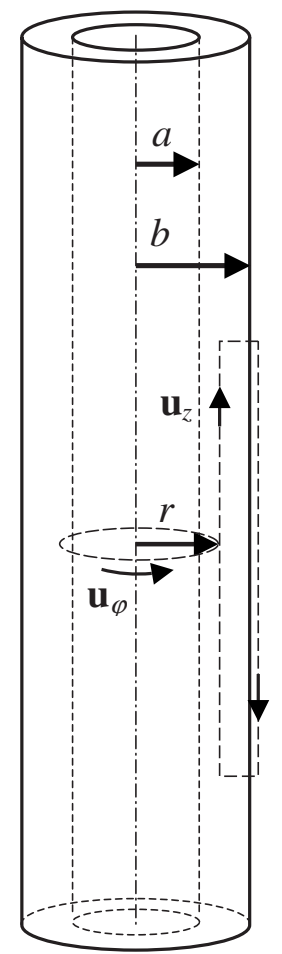

Fig. 1. Schematic diagram of the conductive cylinder showing the circular and rectangular loops for integrating Faraday's induction law and Ampère's circuital law, respectively.

cylindrical geometries. The axial harmonic magnetic field is generated with a long coil, while a short probe coil, located just outside or inside the tube or around the rod, probes the induced field. The induced currents in the probe coil can be used to determine the azimuthal conductivity of the sample.

Precise calculations of eddy currents at high frequencies in tubes and rods are not easy because they involve taking into account the first- and second-order Bessel functions. We have developed a simple approximation, valid at low frequencies, that allows us to obtain very precise values of the azimuthal conductivity of our samples.

At low frequencies, the skin effect is negligible and the circular induced currents are distributed throughout the depth of the conductive cylinder. This ensures that the entire sample is probed. If the tube or rod, normally manufactured by extrusion procedures, has longitudinal flaws, our technique will be able to detect them. Consequently, our approach provides a simple method for nondestructive testing in the field of nonferromagnetic metal manufacturing. ${ }^{1}$

Our technique incorporates basic concepts of electromagnetism and is suitable as a laboratory task for intermediatelevel students in physics and engineering.

\section{BASIC EQUATIONS}

Consider a long nonferrous metal tube with conductivity $\sigma$ and inner and outer radii $a$ and $b$, respectively, as shown in Fig. 1. The tube is subjected to an axial harmonic magnetic field with strength $H(t)=H_{0} \cos \omega t$. In this expression, the amplitude $H_{0}$ is the product of the amplitude of the current in the primary coil and the number of turns per unit length, and $\omega$ is the angular frequency. The induced azimuthal current density at low frequencies can be calculated using Faraday's

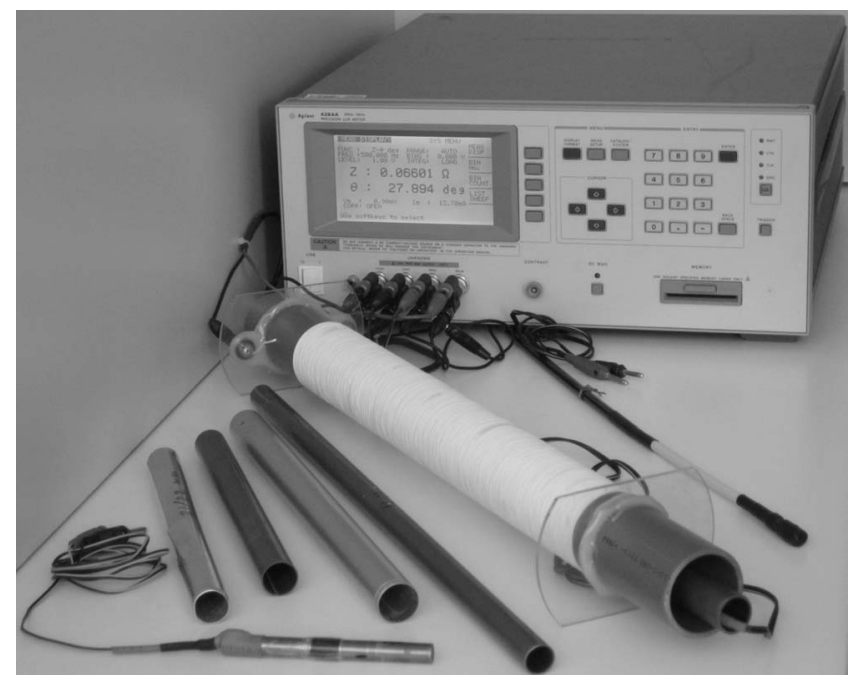

Fig. 2. Photograph of the experimental setup showing the $L C R$ meter, copper and aluminum tubes and rods, and the measuring coils.

induction law $^{2}$ for a circular loop. At a radius $r, a \leq r \leq b$, and neglecting edge effects, the induced electromotive force can be written as

$$
\varepsilon(r, t)=-\frac{d \Phi}{d t}=-\mu_{0} \pi r^{2} \frac{d H(t)}{d t}=\mu_{0} \omega \pi r^{2} H_{0} \sin \omega t
$$

The eddy current in the tube at radius $r$ is equal to

$$
\mathbf{J}(r, t)=\sigma \mathbf{E}(r, t)=\sigma \frac{\varepsilon(r, t)}{2 \pi r} \mathbf{u}_{\phi}=\frac{1}{2} \mu_{0} \sigma \omega r H_{0} \sin \omega t \mathbf{u}_{\phi}
$$

Neglecting the displacement current ${ }^{3}$ and applying Ampère's circuital law for the rectangular loop shown in Fig. 1, we can compute the magnetic flux density. For $a \leq r \leq b$, we find

$$
\mathbf{B}_{\text {tube }}(r, t)=\mu_{0} H_{0}\left[\cos \omega t+\frac{\mu_{0} \sigma \omega}{4}\left(b^{2}-r^{2}\right) \sin \omega t\right] \mathbf{u}_{z} .
$$

For $r \leq a$ the magnetic flux density is uniform,

$$
\mathbf{B}_{\text {in }}(t)=\mu_{0} H_{0}\left[\cos \omega t+\frac{\mu_{0} \sigma \omega}{4}\left(b^{2}-a^{2}\right) \sin \omega t\right] \mathbf{u}_{z} .
$$

The approximate solutions for the eddy currents and the magnetic flux density do not satisfy Maxwell's equations. To obtain more accurate solutions, we must recalculate the current density with the help of the Maxwell-Faraday equation, use Ampère's circuital law, obtain the corresponding correction to the magnetic flux density, etc. After a sufficient number of steps in this iterative process, we reach the desired precision. The successive terms of this chain correspond to the series expansion of the Bessel functions. In the lowfrequency limit, our quasistatic approximation is sufficiently accurate.

The induced voltage in a short coil of $N$ turns, encircling the tube at $r=b$, is equal to 


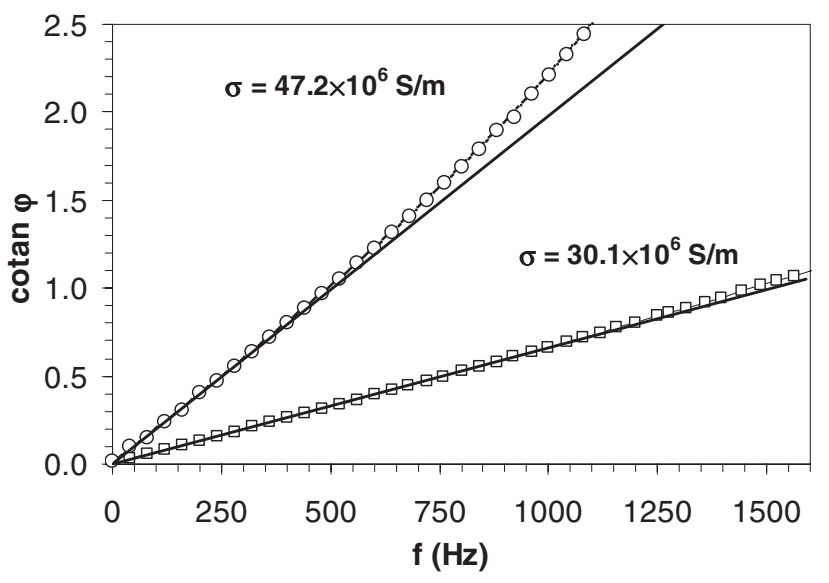

Fig. 3. Results of the measurements of the electrical conductivity in an extruded copper tube (open circles) with inner and outer radii of 10 and 11 $\mathrm{mm}$, respectively, and an aluminum alloy tube (open squares) with inner and outer radii of 10.5 and $11 \mathrm{~mm}$, respectively. The measurements were carried out with a search coil located inside the tube. The straight lines correspond to the low-frequency approximation, while the curves represent the numerical simulation at higher frequencies. The error bars are smaller than the size of the data points.

$$
\begin{aligned}
V_{\text {ext-tube }}(t) & =-\frac{d \Phi_{\text {ext }}}{d t} \\
& =-N \frac{d}{d t}\left[B_{\text {in }}(t) \pi a^{2}+2 \pi \int_{a}^{b} B_{\text {tube }}(r, t) r d r\right] \\
& =\mu_{0} H_{0} N \pi \omega b^{2}\left[\sin \omega t-\frac{\mu_{0} \sigma \omega}{8 b^{2}}\left(b^{4}-a^{4}\right) \cos \omega t\right] .
\end{aligned}
$$

If the probe coil of $N$ turns and cross-sectional area $S$ is located inside the tube, the induced voltage will be

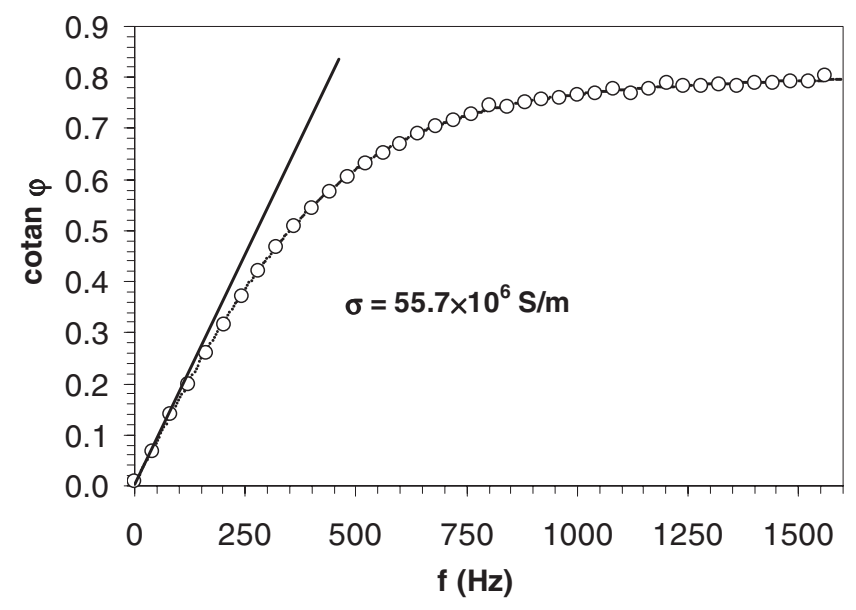

Fig. 4. Results of the measurements of the electrical conductivity in a lathed copper rod with a radius of $5.56 \mathrm{~mm}$. The probe coil used was a monolayer coil of very thin copper wire carefully arranged on the surface of the rod. The straight line corresponds to the low-frequency approximation while the curve represents the numerical simulation at higher frequencies. The error bars are smaller than the size of the data points.

$$
\begin{aligned}
V_{\text {in-tube }}(t) & =-\frac{d \Phi_{\text {in }}}{d t} \\
& =\mu_{0} H_{0} N S \omega\left[\sin \omega t-\frac{\mu_{0} \sigma \omega}{4}\left(b^{2}-a^{2}\right) \cos \omega t\right] .
\end{aligned}
$$

The induced voltage for a nonferromagnetic rod can be obtained from Eq. (5) by setting the inner radius to zero,

$$
V_{\text {rod }}(t)=\mu_{0} H_{0} N \pi b^{2} \omega\left[\sin \omega t-\frac{\mu_{0} \sigma \omega}{8} b^{2} \cos \omega t\right] .
$$

The voltage induced in the probe coil is out-of-phase with respect to the magnetic field strength $H(t)$ by a frequencydependent angle $\varphi$. The phase angles associated with Eqs. (5)-(7) are given by the following expressions:

$$
\begin{aligned}
& \cot \varphi_{\text {ext-tube }}=\frac{\mu_{0} \sigma \omega}{8 b^{2}}\left(b^{4}-a^{4}\right), \\
& \cot \varphi_{\text {in-tube }}=\frac{\mu_{0} \sigma \omega}{4}\left(b^{2}-a^{2}\right), \\
& \cot \varphi_{\text {rod }}=\frac{\mu_{0} \sigma \omega}{8} b^{2} .
\end{aligned}
$$

The conductivity $\sigma$ can thus be determined from a measurement of the phase angle in a similar way to the measurement of the magnetic screening in tubes at low frequencies. ${ }^{4}$

\section{EXPERIMENTAL RESULTS AND EVALUATION}

In this section we describe measurements made at room temperature for a set of samples. The experimental results were obtained by determining the phase angle $\varphi$ using an automatic $L C R$ meter (HP-4284A) operating as a mutual in-

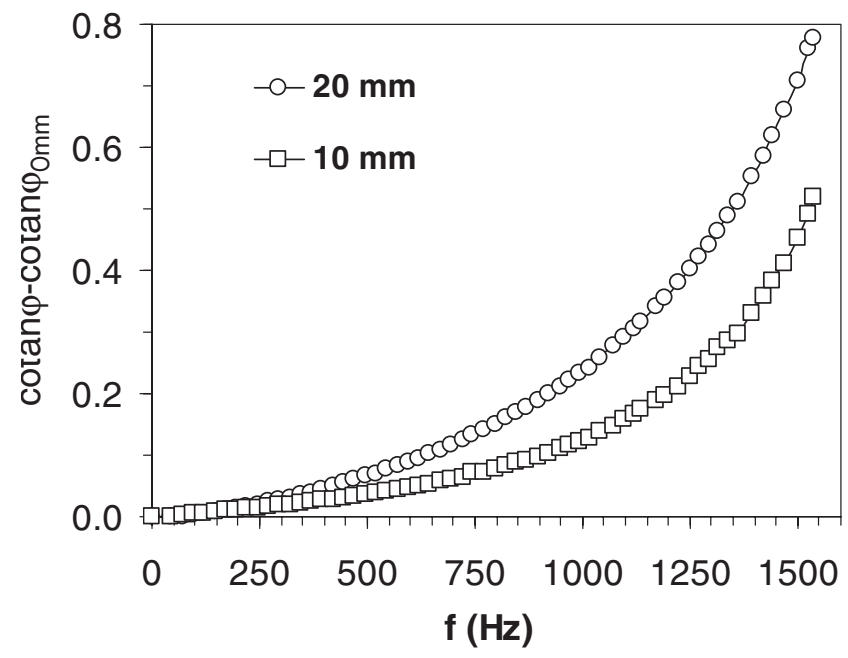

Fig. 5. Results of a defect inspection by means of the electrical conductivity measurement in a copper tube with a $1 \mathrm{~mm}$ diameter pore, using a $1-\mathrm{cm}-$ long probe coil. The differences between the values of $\cot \varphi$ obtained when the probe coil is not centered on the pore and those obtained when the probe coil is centered on the pore are shown as function of the frequency. The open squares and open circles were obtained with the probe coil centered at $10 \mathrm{~mm}$ and $20 \mathrm{~mm}$ from the pore, respectively. The error bars are smaller than the size of the data points. 
ductance bridge, as shown in Fig. 2. The primary coil was connected to the high- and low-current terminals of the $L C R$ meter, and the probe coil was connected to the high- and low-voltage terminals. The $L C R$ meter provides the ratio of the complex quantity $\bar{Z}=\bar{V}_{\text {probe }} / \bar{I}_{\text {primary }}$. The modulus of $\bar{Z}$ is proportional to the mutual inductance modulus, and the argument of $\bar{Z}$ is the phase angle $\varphi$ between $V_{\text {probe }}$ and $I_{\text {primary }}$ To guarantee a stable phase reference in the current and voltage measurement loops, it is convenient to connect the lowvoltage and the low-current terminals of these loops.

We have used a 50-cm-long primary coil with 300 turns and a diameter of $5 \mathrm{~cm}$. The tubes and rods examined are longer than $25 \mathrm{~cm}$. The probe coils have 3000 turns and are about $1 \mathrm{~cm}$ long, and edge effects do not need to be considered. Except for the measurements taken at frequencies of a few hertz, the system operates with excellent resolution and provides stable and repetitive measurements with four significant figures. Because the measurements of the tube geometry were made with a precision of $0.02 \mathrm{~mm}$, we conclude that our procedure provides a conductivity resolution of close to $0.1 \%$. Figure 3 shows the experimental results obtained for extruded copper and aluminum tubes, and Fig. 4 shows the results of our study of a lathed copper rod.

Good accuracy can also be obtained when a two-channel digital oscilloscope or a lock-in amplifier is used. The phase angle can be obtained from the ratio of the amplitudes of the induced voltages before, $V_{0}$, and after, $V_{S}$, the sample has been placed in the system,

$$
\sin \varphi=\frac{V_{0}}{V_{S}} .
$$

In principle, only a true rms voltmeter is required as long as the measurements of $V_{0}$ and $V_{S}$ are carried out with identical currents in the primary coil.

It should be noted that our approach is only valid for driving frequencies up to several hundred hertz. This range is sufficient to perform a very precise measurement of the conductivity of nonferromagnetic cylinders. When the driving frequency increases, the observed relation between $\cot \varphi$ and $\omega$ becomes nonlinear. At these higher frequencies, the skin effect becomes significant and higher-order terms must be included in our calculations to be able to account for the experimental result. The results of finite element calculations with FEMM (Ref. 5) agree with the measurements, as shown in Figs. 3 and 4. The frequency range for which our approximation is valid increases when operating with thinner samples or with materials of lower conductivity, as can be seen for the tube of aluminum in Fig. 3. As an alternative to using a numerical tool to fit the data, we can also use a second-order fit of the measurements and take the slope at the origin to obtain the conductivity in the quasistatic limit.

Because the resolution of our technique is about $0.1 \%$, the temperature must be kept constant during the time required to carry out the measurements. Since the electrical conductivity temperature coefficient of $\mathrm{Cu}$ and $\mathrm{Al}$ is about $0.004 \mathrm{~K}^{-1}$, the temperature fluctuations must be less than $0.25 \mathrm{~K}$ during the measurements. ${ }^{6}$

When the measurements are made using a secondary coil surrounding the tube or rod, it is important to arrange it as a monolayer search coil of very thin copper wire, carefully placed on the surface of the sample. If the measurements were performed using multilayer search coils (larger than the sample radius), the sensitivity of this technique would decrease due to the contribution of the magnetic flux in the region between the coil and the sample to the induced voltage in the secondary coil. This contribution is independent of the presence of the conductive cylinder.

Our simple approximation allows the anisotropic behavior of the electrical conductivity in the extruded tubes to be detected. The data in literature for the conductivity of electrolytic copper range from 55.5 to $58 \mathrm{MS} / \mathrm{m}$ at room temperature, while here we found values of about $47.2 \mathrm{MS} / \mathrm{m}$ for the azimuthal conductivity in the tube (see Fig. 3), clearly showing that the extrusion procedure creates longitudinal microflaws in the wall of the tube. In the case of solid rods, the presence of defects is minimized and our measurements show a conductivity of about 55.7 MS/m (see Fig. 4).

There are abundant references concerning nondestructive testing by means of the eddy current analysis. ${ }^{7,8}$ Our measuring procedure can also be used to study defects in conductive samples. As an example of this possibility, Fig. 5 has been included which shows the results of a defect inspection in a copper tube in the vicinity of a $1 \mathrm{~mm}$ diameter pore. The effective conductivity clearly diminishes when the 1-cm-long probe approaches the pore.

\section{CONCLUSIONS}

We have developed a simple and precise technique to measure the electrical azimuthal conductivity in nonferromagnetic cylinders that can be incorporated into university courses on electromagnetism. The validity of the technique is restricted to low frequencies but its accuracy is noteworthy. It offers a powerful nondestructive technique for the analysis of defects in metallic tubes and is able to detect the anisotropic behavior of electrical conductivity in extruded cylinders.

\section{ACKNOWLEDGMENTS}

The authors would like to thank J. García, laboratory technician at the Department of Applied Physics of the University of Salamanca, for helping manufacture the experimental setup developed for this work. They also acknowledge financial support from the Junta de Castilla y León under Project No. SA114A07 and the Ministerio de Educación y Ciencia of Spain under Project No. MAT-65420-C02-02.

${ }^{1}$ D. J. Hagemaier, Fundamentals of Eddy Current Testing, 7th ed. (The American Society for Nondestructive Testing, Inc., Columbus, $\mathrm{OH}$, 2005).

${ }^{2}$ J. D. Jackson, Classical Electrodynamics, 3rd ed. (Wiley, New York, 1998).

${ }^{3}$ B. D. Popovic, Introductory Engineering Electromagnetic (AddisonWesley, Reading, MA, 1971).

${ }^{4}$ J. Íñiguez, V. Raposo, A. G. Flores, M. Zazo, and A. Hernández-López, "Measurement of the electrical conductivity of metallic tubes by studying magnetic screening at low frequency," Am. J. Phys. 73 (3), 206-210 (2005).

5 FEMM is a freeware Windows-based program for analyzing 2D planar and axisymmetric problems in magnetism via the finite element method with unlimited number of nodes. The package is composed of a triangular 
mesh generator, a conjugate gradient solver and a graphical postprocessor. For more information see $<$ http://femm.foster-miller.net $>$.

${ }^{6}$ Goodfellow, Metals and Materials for Research and Industry, available at

$<$ http://www.goodfellow.com/csp/active/gfHome.csp $>$.

${ }^{7}$ NDT Resource Center, Eddy Current Testing, available at $<$ http://www.ndt-ed.org/EducationResources/HighSchool/Electricity/ eddycurrents.htm $>$.

${ }^{8}$ The American Society for Nondestructive Testing, available at $<$ http://www.asnt.org/publications/materialseval/solution/feb98solution/ feb98sol.htm $>$.

\title{
Contactless measurement of resistivity with DATASTUDIO
}

\author{
Yaakov Kraftmakher ${ }^{\text {a) }}$ \\ Department of Physics, Bar-Ilan University, Ramat-Gan 52900, Israel
}

(Received 14 August 2007; accepted 9 July 2009)

Contactless measurements of the resistivity of nonmagnetic metals are performed automatically with a data-acquisition system. The numerical value of the resistivity appears immediately at the end of data collection. The experiment can be used for student projects. (C) 2009 American Association of Physics Teachers.

[DOI: $10.1119 / 1.3191689]$

The experiment described in this paper demonstrates contactless measurements of the resistivity of nonmagnetic metals. When a conductor is placed in an ac magnetic field, internal eddy currents modify the magnetic field inside the sample. The field can be described in terms of an effective magnetic susceptibility of the sample. This complex quantity depends on the resistivity and shape of the sample and on the frequency of the magnetic field. Under the measurement conditions, the usual magnetic susceptibility of para- and diamagnetic samples is negligible compared to the effective magnetic susceptibility due to the eddy currents in them. The resistivity of a cylindrical sample, positioned in an axial ac magnetic field, can be found from the phase angle of the effective susceptibility. The theory of the measurements is described in Ref. 1 and reproduced in Ref. 2. In some cases, contactless measurements of resistivity may appear to be preferable.

The measurements are performed automatically with a PASCO data-acquisition system and the DATASTUDIO software. A 200 turn coil $L_{1}$ (PASCO EM-6711) forms the primary winding of a differential transformer, as shown in Fig. 1. An 800 turn coil $L_{2}$ (PASCO SF-8611) and a variable resistor $R(20 \Omega)$ are connected in series with $L_{1}$. The sinusoidal output voltage of the signal generator, incorporated into the PASCO SCIENCEWORKSHOP 750 Interface, drives the primary circuit. Coil $L_{2}$ and the 1600 turn coil $L_{3}$ (PASCO SF-8612) form a mutual inductance. The two secondary coils of the differential transformer, $L_{4}$ and $L_{5}$ with 2500 turns each, are $2 \mathrm{~cm}$ in diameter and $2 \mathrm{~cm}$ long. The secondary coils are connected such that without a sample, the output voltage of the differential transformer is zero. When a cylindrical sample is put inside one of the secondary coils, an output voltage appears, whose phase with respect to the phase of the current through the primary coil of the transformer is defined by the phase angle of the effective magnetic susceptibility of the sample. Coils $L_{4}$ and $L_{5}$ are fixed on a common base, whose position inside the primary coil can be adjusted to accurately adjust the output voltage to zero when no sample is present. The setup is similar to the setup used before, ${ }^{2}$ but the coupled coils $L_{2}$ and $L_{3}$ provide an additional voltage, shifted by $90^{\circ}$ compared to the voltage across the resistor $R$. This minor addition allows the phase of the voltage to be measured, which is needed for lock-in detection operation. ${ }^{3,4}$

The output of the differential transformer is connected to the $Y$ input of an oscilloscope (Kenwood CS-4025 model). The voltage across resistor $R$ is connected to the $X$ input. The oscilloscope thus shows the phase relation between the current in the primary coil and the output voltage of the differential transformer due to eddy currents in the sample. The oscilloscope is also used as an amplifier. The voltage at its $Y$ input is amplified and then connected to input $\mathrm{C}$ of the SCIENCEWORKSHOP Interface. The samples used are cylindrical rods made of copper and an aluminum-based alloy. The rods are nearly $1 \mathrm{~cm}$ in diameter and $10 \mathrm{~cm}$ long. Thicker samples are needed to increase the output voltage of the differential transformer.

Using PASCO CI-6503 voltage sensors, the SCIENCEWORKSHOP Interface acquires the voltage across coil $L_{3}$, connected to input $\mathrm{A}$ of the interface, the voltage across resistor $R$, connected to input $\mathrm{B}$, and the amplified output voltage of

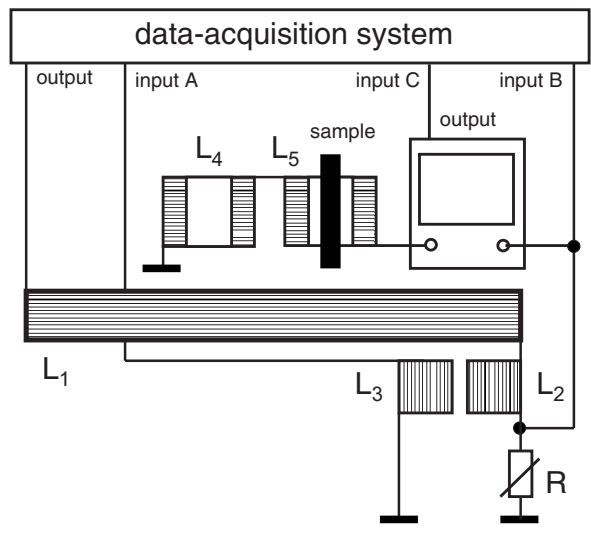

Fig. 1. Schematic diagram of the setup. The secondary coils $L_{4}$ and $L_{5}$ are shown above the primary coil $L_{1}$. 

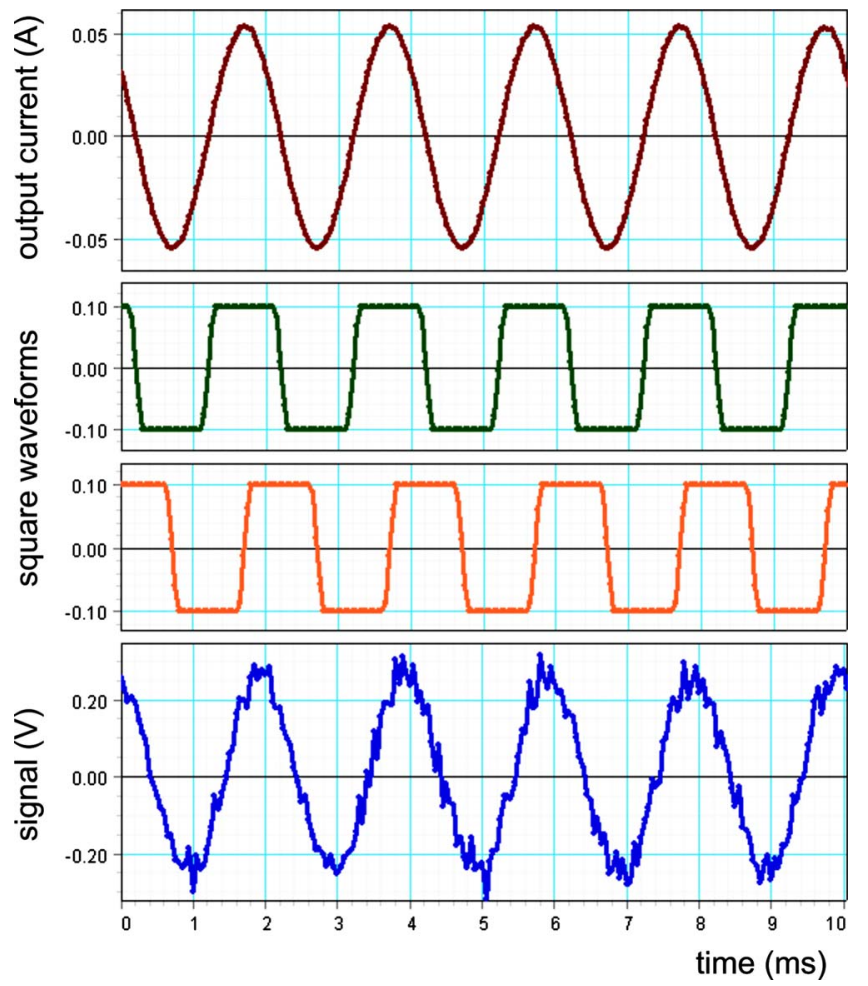

Fig. 2. From top to bottom: output current of the signal generator; two square waveforms derived from the waveforms collected at inputs A and B of the SCIENCEWORKSHOP Interface; and the amplified output signal of the differential transformer when a sample is put into coil $L_{5}$

the differential transformer, connected to input C. Resistor $R$ is adjusted in order to obtain a voltage of amplitude similar to the amplitude of the voltage across coil $L_{3}$; the two voltages are shifted by $90^{\circ}$. Inputs A and B are set to high sensitivity, which restricts the acquired values to less than $0.1 \mathrm{~V}$. As a result, the sinusoidal waveforms at inputs $\mathrm{A}$ and $\mathrm{B}$ are converted into nearly square waveforms, as shown in Fig. 2, which are used in the calculations. When a sine waveform signal is multiplied by a square waveform of fixed amplitude, the time-average of this product is proportional to the amplitude of the sine waveform signal and to the cosine of the phase shift between the two waveforms; this principle is used in lock-in detectors.

During data collection, the Calculate tool of DATASTUDIO continuously computes the following quantities:

(1) $Y_{1}=V_{A^{\prime}} \times V_{C}$ and $Y_{2}=V_{B^{\prime}} \times V_{C}$, where $V_{C}$ is the voltages at input $\mathrm{C}$. The voltages $V_{A^{\prime}}$ and $V_{B^{\prime}}$ are the nearly square waveforms, derived from the voltages at inputs A and $\mathrm{B}$.

(2) $Y_{3}$ and $Y_{4}$, the time-averaged values of $Y_{1}$ and $Y_{2} . Y_{3}$ and $Y_{4}$ are proportional to $\sin \alpha$ and $\cos \alpha$, where $\alpha$ is an angle complementary to the phase angle of the effective magnetic susceptibility of the sample. These operations are similar to those of a dual-channel lock-in detector.

(3) $z=\tan \alpha=Y_{3} / Y_{4}$. The value of $z$, which is independent of the amplitude of the output voltage of the differential transformer, is sufficient to determine the resistivity of the sample.

(4) The resistivity of the sample (see Ref. 2),
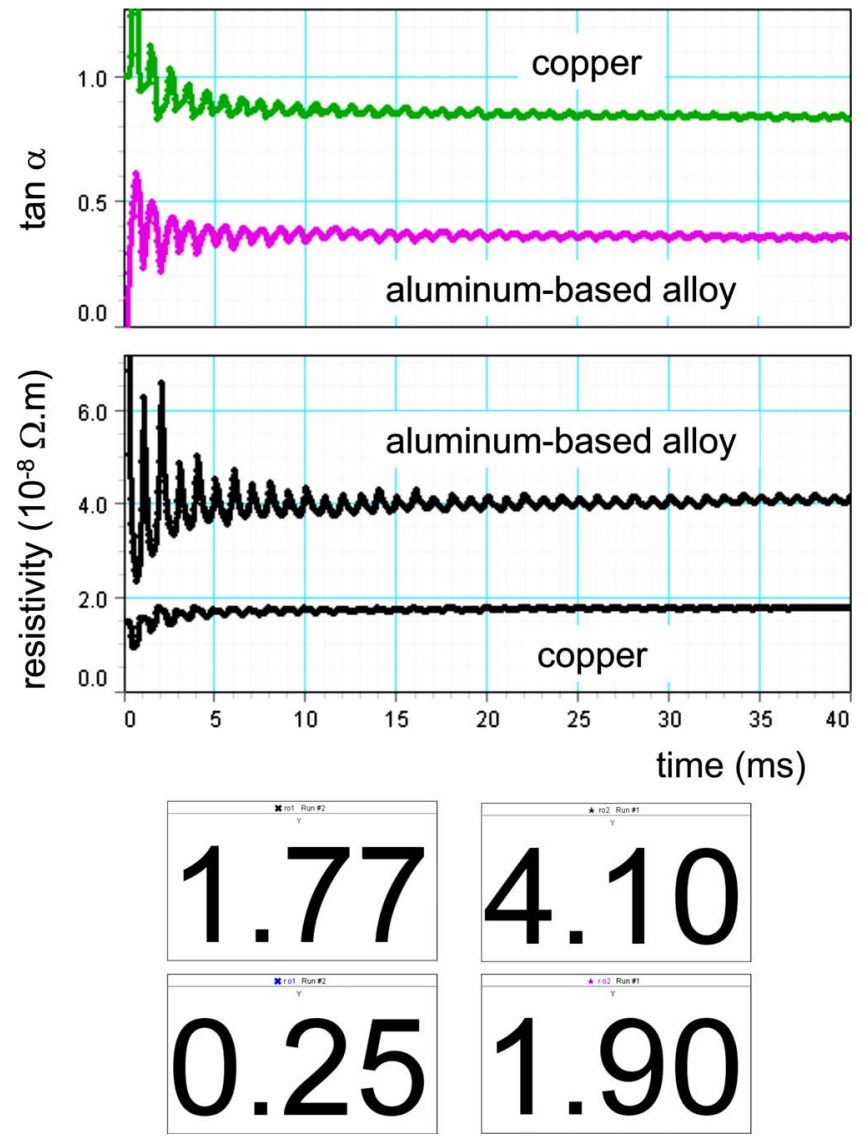

Fig. 3. The averaging process and digital displays of the resistivity of copper and an aluminum-based alloy at room and liquid nitrogen temperatures.

$$
\begin{aligned}
\rho\left(10^{-8} \Omega \mathrm{m}\right)= & 395^{*} a^{2} f /\left(-0.01+3.06^{*} z-0.105^{*} z^{\wedge} 2\right. \\
& \left.+0.167^{*} z^{\wedge} 3\right),
\end{aligned}
$$

where $a$ is the radius of the sample (in $\mathrm{m}$ ) and $f$ is the frequency of the magnetic field (in $\mathrm{Hz}$ ). The diameter of the sample and the frequency of the magnetic field are defined with the Calculate tool window.

All operations of DATASTUDIO are readily observable. An example of the last two operations is shown in Fig. 3.

At room temperature, the frequency of the magnetic field used is $500 \mathrm{~Hz}$. The sampling rate is $20 \mathrm{kHz}$ and data collection is stopped after $40 \mathrm{~ms}$. At the end of data collection, the resistivity is displayed in digital form. For measurements at liquid nitrogen temperature, a dewar, filled with liquid nitrogen, is placed inside the primary coil and the secondary coils are immersed in the liquid. At these temperatures, the frequency of the magnetic field used is $200 \mathrm{~Hz}$.

The resistivities obtained are in reasonable agreement with existing data. The accepted resistivity of pure copper is $1.70 \times 10^{-8} \Omega \mathrm{m}$ at $297 \mathrm{~K}$ and $0.19 \times 10^{-8} \Omega \mathrm{m}$ at $77 \mathrm{~K}^{5}$ The resistivities obtained in our measurements, $1.77 \times 10^{-8} \Omega \mathrm{m}$ at $297 \mathrm{~K}$ and $0.25 \times 10^{-8} \Omega \mathrm{m}$ at $77 \mathrm{~K}$, are somewhat higher probably due to the contributions of impurities and physical imperfections in our sample. These contributions should be added to the resistivity of the perfect sample and depend weakly on temperature. The temperature dependence of the resistivity of the aluminum-based alloy is 
much weaker than that of pure metals. For Al-Si alloys, the accepted resistivities at $297 \mathrm{~K}$ are in the range from $3.85 \times 10^{-8}$ to $4.25 \times 10^{-8} \Omega \mathrm{m}$, depending on the concentration of silicon. $^{6}$ The measured resistivity of $4.10 \times 10^{-8} \Omega \mathrm{m}$ at $297 \mathrm{~K}$ is in excellent agreement with the range of accepted values.

The experiment is relevant to some important topics in electromagnetism such as eddy currents, skin effect, effective magnetic susceptibility, and resistivity of metals and alloys. It can also serve as an illustration of lock-in detection. The DATASTUDIO setup file for the experiment is available online. $^{7}$
${ }^{a)}$ Electronic mail: krafty@mail.biu.ac.il

${ }^{1}$ R. G. Chambers and J. G. Park, "Measurement of electrical resistivity by a mutual inductance method," Br. J. Appl. Phys. 12, 507-510 (1961).

${ }^{2}$ Y. Kraftmakher, "Eddy currents: Contactless measurement of electrical resistivity," Am. J. Phys. 68, 375-379 (2000).

${ }^{3}$ Y. Kraftmakher, "Lock-in detection with DATASTUdio," Am. J. Phys. 74, 207-210 (2006)

${ }^{4}$ Y. Kraftmakher, Experiments and Demonstrations in Physics. Bar-Ilan Physics Laboratory (World Scientific, Singapore, 2007).

${ }^{5}$ G. K. White and M. L. Minges, "Thermophysical properties of some key solids: An update," Int. J. Thermophys. 18, 1269-1327 (1997).

${ }^{6}$ R. Brandt and G. Neuer, "Electrical resistivity and thermal conductivity of pure aluminum and aluminum alloys up to and above the melting temperature," Int. J. Thermophys. 28, 1429-1446 (2007).

${ }^{7}<$ http://www.ph.biu.ac.il/data/teach/h/86-232/BIU_Lab.html>.

\title{
APS/AAPT Online Journal: Physical Review Special Topics - Physics Education Research
}

A peer-reviewed, electronic journal exploring experimental and theoretical research on the teaching and learning of physics.

\author{
Edited by: \\ Robert Beichner \\ North Carolina State University
}

Access Is Free!

Features:

- review articles

- methodology critiques

- new assessment tools and research techniques

To learn more about the PER journal visit: http://www.aapt.org/Publications/perjournal.cfm

Physical Review Special Topics-Physics Education Research is sponsored by APS, AAPT, and the APS Forum on Education. 